\title{
Representations of Higher Adelic Groups and Arithmetic *
}

\author{
A. N. Parshin ${ }^{\dagger}$
}

What do we mean by local ? To get an answer to this question let us start from the following two problems.

First problem is from number theory. When does the diophantine equation

$$
f(x, y, z)=x^{2}-a y^{2}-b z^{2}=0, \quad a, b, \in \mathbb{Q}^{*}
$$

have a non-trivial solution in rational numbers ? In order to solve the problem, let us consider the quadratic norm residue symbol $(-,-)_{p}$ where $p$ runs through all primes $p$ and also $\infty$. This symbol is a bi-multiplicative map $(-,-)_{p}: \mathbb{Q}^{*} \times \mathbb{Q}^{*} \rightarrow\{ \pm 1\}$ and it is easily computed in terms of the Legendre symbol. Then, a non-trivial solution exists if and only if, for any $p,(a, b)_{p}=1$. However, these conditions are not independent:

$$
\prod_{p}(a, b)_{p}=1
$$

This is essentially the Gauss reciprocity law in the Hilbert form.

The "points" $p$ correspond to all possible completions of the field $\mathbb{Q}$ of rational numbers, namely to the $p$-adic fields $\mathbb{Q}_{p}$ and the field $\mathbb{R}$ of real numbers. One can show that the equation $f=0$ has a non-trivial solution in $\mathbb{Q}_{p}$ if and only if $(a, b)_{p}=1$.

The second problem comes from complex analysis. Let $X$ be a compact Riemann surface $(=$ complete smooth algebraic curve defined over $\mathbb{C})$. For a point $P \in X$, denote by $K_{P}=\mathbb{C}\left(\left(t_{P}\right)\right)$ the field of Laurent formal power series in a local coordinate $t_{P}$ at the point $P$. The field $K_{P}$ contains the ring $\widehat{\mathcal{O}}_{P}=\mathbb{C}\left[\left[t_{P}\right]\right]$ of Taylor formal power series. These have an invariant meaning and are called the local field and the local ring at $P$ respectively. Let us now fix finitely many points $P_{1}, \ldots, P_{n} \in X$ and assign to every $P$ in $X$ some elements $f_{P}$ such that $f_{P_{1}} \in K_{P_{1}}, \ldots, f_{P_{n}} \in K_{P_{n}}$ and $f_{P}=0$ for all other points.

*To appear in Proceedings of the International Congress of Mathematicians, Hyderabad, India, 2010, vol. 1 .

†Steklov Mathematical Institute, Russian Academy of Sciences, Gubkina str 8, 119991 Moscow, Russia. I am very thankful to Lawrence Breen, Denis V. Osipov, Vladimir L. Popov and Yuri G. Zarhin who have read the text and made many valuable remarks. The author was supported by RFBR (grant no. 08-01-00095-a), by a program for supporting Leading Scientific Schools (grant no. Nsh-4713.2010.1 ) . 
When does there exist a meromorphic (=rational) function $f$ on $X$ such that

$$
f_{P}-f \in \widehat{\mathcal{O}}_{P} \quad \text { for every } P \in X ?
$$

The classical answer to this Cousin problem is the following: there exists such an $f$ whenever for any regular differential form $\omega$ on $X$

$$
\sum_{P} \operatorname{res}_{P}\left(f_{P} \omega\right)=0
$$

The space of regular differential forms has dimension $g(=$ genus of $X$ ) and in this way one gets finitely many conditions on the data $\left(f_{P}\right)$. The residue is an additive map $\operatorname{res}_{P}: \Omega^{1}\left(K_{P}\right) \rightarrow \mathbb{C}$ and is easily computed in terms of the local decomposition of the differential form $\omega \in \Omega^{1}\left(K_{P}\right)$. Note that "locally", problem (2) can be solved for any point $P$. Behind our global conditions (3), we have the following residue relation:

$$
\sum_{P} \operatorname{res}_{P}(\eta)=0
$$

for any meromorphic differential form $\eta$ on $X$.

We see some similarity between these two problems, which belong to very different parts of our science. The explanation lies in the existence of a very deep analogy between numbers and functions, between number fields and fields of algebraic functions. This analogy goes back to the nineteenth century, possibly to Kronecker. The leading role in the subsequent development belongs to Hilbert. The analogy was one of his beloved ideas, and thanks to Hilbert it became one of the central ideas in the development of number theory during the twentieth century. Following this analogy, we can compare algebraic curves over $\mathbb{C}$ (= compact Riemann surfaces) and number fields (= finite extensions of $\mathbb{Q})$. In particular, this includes a comparison of local fields such as that between the fields $\mathbb{C}((t))$ and $\mathbb{Q}_{p}$. Their similarity was already pointed out by Newton 1 .

In modern terms, we have two kinds of geometric objects. First, a complete algebraic curve $X$, containing an affine curve $U=\operatorname{Spec}(R)_{2}^{2}$, where $R$ is the ring of regular functions on $U$ :

$$
\text { (geometric picture) } X \supset U \text { and finitely many points } P \in X \text {. }
$$

Next, if we turn to arithmetic, we have a finite extension $K \supset \mathbb{Q}$ and the ring $R \subset K$ of integers. We write

(arithmetic picture) $\quad X \supset U=\operatorname{Spec}(R)$ and finitely many infinite places $P \in X$.

The places ("points") correspond to the embeddings of $K$ into the fields $\mathbb{R}$ or $\mathbb{C}$. Here, $X$ stands for the as yet not clearly defined complete "arithmetical" curve, an analogue of the curve $X$ in the geometric situation. The analogy between both $U$ 's is very clear

\footnotetext{
${ }^{1} \mathrm{He}$ compared the power series and the expansions of rational numbers in powers of $p$ (for $p=10$ ).

${ }^{2}$ Here, $\operatorname{Spec}(R)$ is the set of prime ideals in the ring $R$ together with the additional structure of a scheme.
} 
and transparent. The rings $R$ are the Dedekind rings of the Krull dimension ${ }^{3} 1$. The nature of the additional points (outside $U$ ) are more complicated. In the geometric case, they also correspond to the non-archimedean valuations on the curve $X$, whereas in the arithmetical case these infinite places are a substitute for the archimedean valuations of the field $K$.

In algebraic geometry, we also have the theory of algebraic curves defined over a finite field $\mathbb{F}_{q}$ and this theory, being arithmetic in its nature, is much closer to the theory of number fields than the theory of algebraic curves over $\mathbb{C}$. The main construction on both sides of the analogy is the notion of a local field. These local fields appear into the following table:

\begin{tabular}{|c|c|c|}
\hline $\begin{array}{c}\text { dimension } \\
>2\end{array}$ & $\begin{array}{c}\text { geometric case } \\
\ldots\end{array}$ & $\begin{array}{c}\text { arithmetic case } \\
\ldots\end{array}$ \\
\hline 2 & $?$ & $\mathbb{R}((t)), \mathbb{C}((t))$ \\
\hline 1 & $\mathbb{F}_{q}((t))$ & $\mathbb{Q}_{p}, \mathbb{R}, \mathbb{C}$ \\
\hline 0 & $\mathbb{F}_{q}$ & $\mathbb{F}_{1}$ \\
\hline
\end{tabular}

Here $\mathbb{F}_{1}$ is the so-called "field" with one element, which is quite popular nowadays. We will see soon why the fields $\mathbb{R}((t))$ and $\mathbb{C}((t))$ belong to the higher level of the table than the fields $\mathbb{Q}_{p}$ or $\mathbb{R}$. More on the analogy between geometry and arithmetic can be found in 61.

\section{1 n-dimensional Local Fields and Adelic Groups}

Let us consider algebraic varieties $X$ (or Grothendieck schemes) of dimension greater than one. It appears that we have a well established notion of something local attached to a point $P \in X$. One can take a neighborhood of $P$, e.g. affine, complex-analytic if $X$ is defined over $\mathbb{C}$, formal and so on. In this talk we will advocate the viewpoint that the genuine local objects on the varieties are not the points with some neighborhoods but the maximal ordered sequences (or flags) of subvarieties, ordered by inclusion.

If $X$ is a variety (or a scheme) of dimension $n$ and

$$
X_{0} \subset X_{1} \subset \ldots X_{n-1} \subset X_{n}=X
$$

is a flag of irreducible subvarieties $\left(\operatorname{dim}\left(X_{i}\right)=i\right)$ then one can define a certain ring

$$
K_{X_{0}, \ldots, X_{n-1}}
$$

associated to the flag. In the case where all the subvarieties are regularly embedded, this ring is an $n$-dimensional local field.

Definition 1. Let $K$ and $k$ be fields. We say that $K$ has a structure of an $n$ dimensional local field with the last residue field $k$ if either $n=0$ and $K=k$ or $n \geq 1$

\footnotetext{
${ }^{3}$ That is, the length of a maximal chain of prime ideals. The ring $R$ itself is not a prime ideal.
} 
and $K$ is the fraction field of a complete discrete valuation ring $\mathcal{O}_{K}$ whose residue field $\bar{K}$ is a local field of dimension $n-1$ with the last residue field $k$.

Thus, an $n$-dimensional local field has the following inductive structure:

$$
K=: K^{(0)} \supset \mathcal{O}_{K} \rightarrow \bar{K}=: K^{(1)} \supset \mathcal{O}_{\bar{K}} \rightarrow \bar{K}^{(1)}=: K^{(2)} \supset \mathcal{O}_{K^{(2)}} \rightarrow \ldots \rightarrow \bar{K}^{(n)}=k
$$

where $\mathcal{O}_{F}$ denotes the valuation ring of the valuation on $F$ and $\bar{F}$ denotes the residue field.

The simplest example of an $n$-dimensional local field is the field

$$
K=k\left(\left(t_{1}\right)\right)\left(\left(t_{2}\right)\right) \ldots\left(\left(t_{n}\right)\right)
$$

of iterated Laurent formal power series. In dimension one, there are examples from the table. However, fields such as $\mathbb{R}$ or $\mathbb{C}$ are not covered by this definition. Concerning classification of the local fields see [17].

One can then form the adelic group (actually, the ring)

$$
\mathbb{A}_{X}=\prod^{\prime} K_{X_{0}, \ldots, X_{n-1}}
$$

where the product is taken over all the flags with respect to certain restrictions on components of adeles. For schemes over a finite field $\mathbb{F}_{q}$, this is the ultimate definition of the adelic space attached to $X$. In general, one must extend it by adding archimedean components, such as the fields $\mathbb{R}$ or $\mathbb{C}$ in dimension one.

In dimension one, the local fields and adelic groups are well-known tools of arithmetic. They were introduced by C. Chevalley in the 1930s and were used to formulate and solve many problems in number theory and algebraic geometry (see, for example, [1, 74]). These constructions are associated with fields of algebraic numbers and fields of algebraic functions in one variable over a finite field, that is with schemes of dimension 1. A need for such constructions in higher dimensions was realized by the author in the 1970s. They were developed in the local case for any dimension and in the global case for dimension two [53, 54, 17, 58. This approach was extended by A. A. Beilinson to the schemes of an arbitrary dimension [3, 25]. In this talk, we restrict ourselves to the case of dimension two.

Let $X$ be a smooth irreducible surface over a field $k$ (or an arithmetic surface), let $P$ be a closed point of $X$ and let $C \subset X$ be an irreducible curve such that $P \in C$. We denote by $\mathcal{O}_{X, P}$ the local ring at the point $P$, that is the ring of rational functions which are regular at $P$. We denote also by $\mathcal{O}_{C}$ the ring of rational functions on $X$ which have no pole along the $C$.

If $X$ and $C$ are smooth at $P$, then we pick a local equation $t \in \mathcal{O}_{X, P}$ of $C$ at $P$ and choose $u \in \mathcal{O}_{X, P}$ such that $\left.u\right|_{C} \in \mathcal{O}_{C, P}$ is a local parameter at $P$. Denote by $\wp$ the ideal in $\mathcal{O}_{X, P}$ defining the curve $C$ near $P$. We can introduce a two-dimensional local field $K_{P, C}$ attached to the pair $P, C$ by the following procedure which includes completions 
and localizations:

$$
\begin{array}{ccc}
\widehat{\mathcal{O}}_{X, P} & = & k(P)[[u, t]] \supset \wp=(t) \\
\widehat{\mathcal{O}}_{X, P}^{\mid} & = & \text {discrete valuation ring with residue field } k(P)((u)) \\
\left(\widehat{\mathcal{O}}_{X, P}^{\mid}\right)_{\wp} & k(P)((u))[[t]] \\
\widehat{\mathcal{O}}_{P, C}:={\widehat{\left(\widehat{\mathcal{O}}_{X, P}\right)_{\wp}}}_{\mid}= & k(P)((u))((t))
\end{array}
$$

Note that the left-hand construction is meaningful even without smoothness of the curve $C$ (it is sufficient to assume that $C$ has only one formal branch near $P$ ). In the general case, the ring $K_{P, C}$ is a finite direct sum of 2-dimensional local fields. If $P$ is smooth then the field $K_{P, C}$ has the following informal interpretation. Take a function $f$ on $X$. We can, first, develop $f$ as a formal power series in the variable $t$ along the curve $C$ and then every coefficient of the series restricted to $C$ can be further developed as a formal power series in the variable $u$. The local field $K_{P, C}$ is a kind of completion of the field of rational functions $K=k(X)$ on $X$. It carries a discrete valuation $\nu_{C}: K_{P, C}^{*} \rightarrow \mathbb{Z}$ defined by the powers of the ideal $\wp$.

Let $K_{P}$ be the minimal subring of $K_{P, C}$ which contains both $k(X)$ and $\widehat{\mathcal{O}}_{X, P}$. In general, the ring $K_{P}$ is not a field. Then $K \subset K_{P} \subset K_{P, C}$ and there is another intermediate subring $K_{C}=\operatorname{Frac}\left(\widehat{\mathcal{O}}_{C}\right) \subset K_{P, C}$. We can compare the structure of the local adelic components in dimensions one and two:

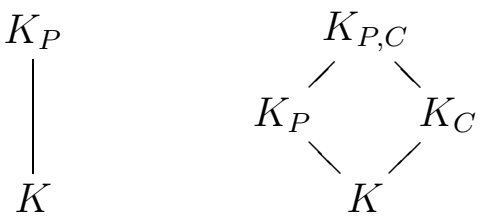

The global adelic group is a certain subgroup of the ordinary product of all twodimensional local fields. Namely, a collection $\left(f_{P, C}\right)$ where $f_{P, C} \in K_{P, C}$ belongs to $\mathbb{A}_{X}$ if the following two conditions are satisfied:

$$
\left\{f_{P, C}\right\} \in \mathbb{A}_{C}\left(\left(t_{C}\right)\right)
$$

for a fixed irreducible curve $C \subset X$ and a local equation $t_{C}=0$ of the curve $C$ on some open affine subset $U \subset X$ and

- we have $\nu_{C}\left(f_{P, C}\right) \geq 0$, or equivalently

$$
\left\{f_{P, C}\right\} \in \mathbb{A}_{C}\left[\left[t_{C}\right]\right]
$$

for all but finitely many irreducible curves $C \subset X$. 
Here we reduced the definition of the adelic group to the classical case of algebraic curves $C$. Recall that a collection $\left(f_{P}, P \in C\right)$ belongs to the adelic (or restricted) product $\mathbb{A}_{C}$ of the local fields $K_{P}$ if and only if for almost all points $P$ we have $f_{P} \in \widehat{\mathcal{O}}_{P}$.

What can one do with this notion of the local field and why is it really local ? To get some understanding of this, we would like to develop the above examples (of residues and symbols) in dimension two. For any flag $P \in C$ on a surface $X$ and a rational differential form $\omega$ of degree 2 we can define the residue

$$
\operatorname{res}_{P, C}(\omega)=\operatorname{Tr}_{k(P) / k}\left(a_{-1,-1}\right)
$$

where $\omega=\sum_{i, j} a_{i, j} u^{i} t^{j} d u \wedge d t$ in the field $K_{P, C} \cong k(P)((u))((t))$. Then, instead of the simple relation (4) on an algebraic curve, we get two types of relations on the projective surface $X[54$

$$
\begin{aligned}
& \sum_{P \in C} \operatorname{res}_{P, C}(\omega)=0, \quad \text { for any fixed curve } C, \\
& \sum_{C \ni P} \operatorname{res}_{P, C}(\omega)=0, \quad \text { for any fixed point } P .
\end{aligned}
$$

At the same time, we can define certain symbols (bi-multiplicative and threemultiplicative) 53

$$
(-,-)_{P, C}: K_{P, C}^{*} \times K_{P, C}^{*} \rightarrow \mathbb{Z} \text { and }(-,-,-)_{P, C}: K_{P, C}^{*} \times K_{P, C}^{*} \times K_{P, C}^{*} \rightarrow k^{*}
$$

which are respectively generalizations of the valuation $\nu_{P}: K_{P}^{*} \rightarrow \mathbb{Z}$ and the norm residue symbol $(-,-)_{P}: K_{P}^{*} \times K_{P}^{*}$ (actually, the tame symbol) on an algebraic curve $C$. The reciprocity laws have the same structure as the residue relations. In particular, if $f, g, h \in K^{*}$ then

$$
\begin{aligned}
& \prod_{P \in C}(f, g, h)_{P, C}=1, \quad \text { for any fixed curve } C, \\
& \prod_{C \ni P}(f, g, h)_{P, C}=1, \quad \text { for any fixed point } P .
\end{aligned}
$$

This shows that in dimension two there is a symmetry between points $P$ and curves $C$ (which looks like the classical duality between points and lines in projective geometry).

If $C$ is a curve then the space $\mathbb{A}_{C}$ contains the important subspaces $\mathbb{A}_{0}=K=k(C)$ of principal adeles (rational functions diagonally embedded into the adelic group) and $\mathbb{A}_{1}=\prod_{P \in C} \widehat{\mathcal{O}}_{P}$ of integral adeles. These give rise to the adelic complex

$$
\mathbb{A}_{0} \oplus \mathbb{A}_{1} \rightarrow \mathbb{A}_{C}
$$

This complex computes the cohomology of the structure sheaf $\mathcal{O}_{C}$. If $D$ is a divisor on $C$ then the cohomology of the sheaf $\mathcal{O}_{C}(D)$ can be computed using the adelic complex (17) where the subgroup $\mathbb{A}_{1}$ is replaced by the subgroup $\mathbb{A}_{1}(D)=\left\{\left(f_{P}\right) \in A_{C}: \nu_{P}\left(f_{P}\right)+\right.$ $\nu_{P}(D)>0$ for any $\left.P \in C\right\}$.

In dimension two, there is a much more complicated structure of subspaces in $\mathbb{A}_{X}$ (see [58]). Among the others, it includes three subspaces $\mathbb{A}_{12}=\prod_{P \in C}^{\prime} \widehat{\mathcal{O}}_{P, C}, \mathbb{A}_{01}=\prod_{C \subset X}^{\prime} K_{C}$ 
and $\mathbb{A}_{02}=\prod_{P \in X}^{\prime} K_{P}$. We set $\mathbb{A}_{0}=\mathbb{A}_{01} \cap \mathbb{A}_{02}, \mathbb{A}_{1}=\mathbb{A}_{01} \cap \mathbb{A}_{12}$ and $\mathbb{A}_{2}=\mathbb{A}_{02} \cap \mathbb{A}_{12}$, and arrive at an adelic complex

$$
\mathbb{A}_{0} \oplus \mathbb{A}_{1} \oplus \mathbb{A}_{2} \rightarrow \mathbb{A}_{01} \oplus \mathbb{A}_{02} \oplus \mathbb{A}_{12} \rightarrow \mathbb{A}_{X}
$$

Once again, the complex computes the cohomology of the sheaf $\mathcal{O}_{X}$. One can extend these complexes to the case of arbitrary schemes $X$ and any coherent sheaf on $X$ (see [3, 25, 17]).

The last issue which we will discuss in this section is the relation between the residues and Serre duality for coherent sheaves. We will only consider the construction of the fundamental class for the sheaf of differential forms. For curves $C$, we have an isomorphism $H^{1}\left(C, \Omega_{C}^{1}\right) \cong \Omega^{1}\left(\mathbb{A}_{C}\right) / \Omega^{1}\left(\mathbb{A}_{0}\right) \oplus \Omega^{1}\left(\mathbb{A}_{1}\right)$. The fundamental class isomorphism $H^{1}\left(C, \Omega_{C}^{1}\right) \cong k$ can be defined as the sum of residues on $\Omega^{1}\left(\mathbb{A}_{C}\right)$. The residues relation (3) shows that this sum vanishes on the subspace $\Omega^{1}\left(\mathbb{A}_{0}\right)$ (and it vanishes on the other subspace $\Omega^{1}\left(\mathbb{A}_{1}\right)$ for trivial reasons). The same reasoning works in the case of surfaces. We have an isomorphism

$$
H^{2}\left(C, \Omega_{X}^{2}\right) \cong \Omega^{2}\left(\mathbb{A}_{X}\right) / \Omega^{2}\left(\mathbb{A}_{01}\right) \oplus \Omega^{2}\left(\mathbb{A}_{02}\right) \oplus \Omega^{2}\left(\mathbb{A}_{12}\right) \rightarrow k
$$

where the last arrow is again the sum of residues over all flags $P \in C \subset X$. The correctness of this definition follows from the residues relations (5) and (6) . We refer to [54, 3, 17] for the full description of the duality.

\section{Harmonic Analysis on Two-dimensional Schemes}

In the 1-dimensional case, local fields and adelic groups both carry a natural topology for which they are locally compact groups and classical harmonic analysis on locally compact groups can therefore be applied to this situation. The study of representations of algebraic groups over local fields and adelic groups is a broad subfield of representation theory, algebraic geometry and number theory. Even for abelian groups, this line of thought has very nontrivial applications in number theory, particularly to the study of L-functions of one-dimensional schemes (see below). The first preliminary step is the existence of a Haar measure on locally compact groups. The analysis starts with a definition of certain function spaces.

We have two sorts of locally compact groups. The groups of the first type are totally disconnected such as the fields $\mathbb{Q}_{p}$ or $\mathbb{F}_{q}((t))$. These groups are related with varieties defined over a finite field. The groups of the second type are connected Lie groups such as the fields $\mathbb{R}$ or $\mathbb{C}$.

If $V$ is a locally compact abelian group of the first type let us consider the following spaces of functions (or distributions) on $V$ :

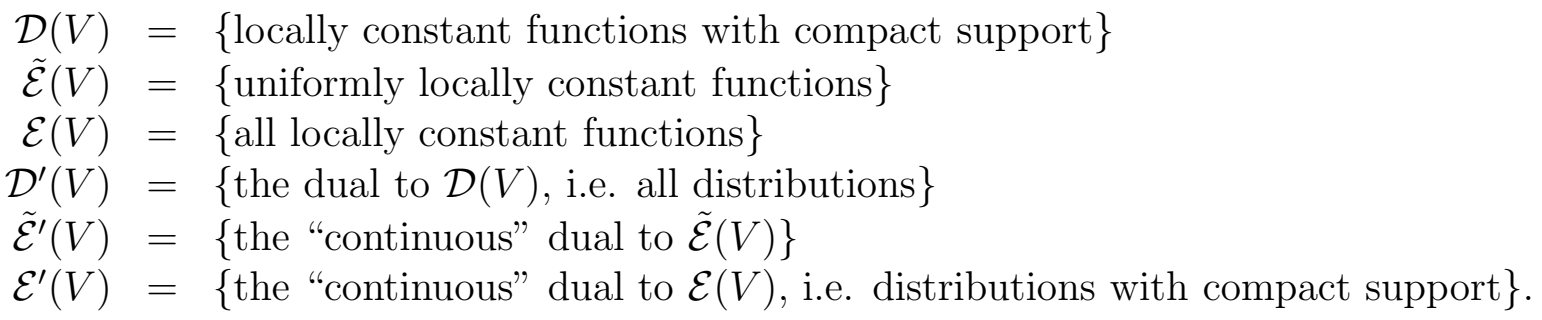


These are the classical spaces introduced by F. Bruhat [10] and the more powerful way to develop the harmonic analysis is the categorical point of view. First, we need definitions of direct and inverse images with respect to the continuous homomorphisms.

Let $f: V \rightarrow W$ be a strict homomorphism 4 of locally compact groups $V$ and $W$. Then the inverse image $f^{*}: \mathcal{D}(W) \rightarrow \mathcal{D}(V)$ is defined if and only if the kernel of $f$ is compact. The direct image $f_{*}: \mathcal{D}(V) \otimes \mu(V) \rightarrow \mathcal{D}(W)$ is defined if and only if the cokernel of $f$ is discrete. Here, $\mu(V)$ is a (1-dimensional) space of Haar measures on $V$. For the spaces like $\mathcal{E}, \tilde{\mathcal{E}}$ the inverse image is defined for any $f$, but the direct image is defined if and only if the kernel is compact and the cokernel is discrete. For the distribution spaces the corresponding conditions are the dual ones. Therefore, we see that these maps do not exist for arbitrary homomorphisms in our category and there are some "selection rules".

The Fourier transform $\mathrm{F}$ is defined as a map from $\mathcal{D}(V) \otimes \mu(V)$ to $\mathcal{D}(\check{V})$ as well as for the other types of spaces. Here, $\check{V}$ is the dual group. The main result is the following Poisson formula

$$
\mathrm{F}\left(\delta_{W, \mu_{0}} \otimes \mu\right)=\delta_{W^{\perp}, \mu^{-1} / \mu_{0}^{-1}}
$$

for any closed subgroup $i: W \rightarrow V$. Here $\mu_{0} \in \mu(W) \subset \mathcal{D}^{\prime}(W), \mu \in \mu(V) \subset$ $\mathcal{D}^{\prime}(V), \delta_{W, \mu_{0}}=i_{*}\left(1_{W} \otimes \mu_{0}\right)$ and $W^{\perp}$ is the annihilator of $W$ in $\check{V}$.

This general formula is very efficient when applied to the self-dual (!) group $\mathbb{A}_{C}$. The standard subgroups in $\mathbb{A}_{C}$ have their characteristic functions $\delta_{\mathbb{A}_{1}(D)} \in \mathcal{D}\left(\mathbb{A}_{C}\right)$ and $\delta_{K} \in \mathcal{D}^{\prime}\left(\mathbb{A}_{C}\right)$. We have

$$
\begin{gathered}
\mathrm{F}\left(\delta_{\mathbb{A}_{1}(D)}\right)=\operatorname{vol}\left(\mathbb{A}_{1}(D)\right) \delta_{\mathbb{A}_{1}((\omega)-D)}, \\
\mathrm{F}\left(\delta_{K}\right)=\operatorname{vol}\left(\mathbb{A}_{C} / K\right)^{-1} \delta_{K},
\end{gathered}
$$

where $K=\mathbb{F}_{q}(C)$ and $(\omega)$ is the divisor of a nonzero rational differential form $\omega \in \Omega_{K}^{1}$ on $C$. There is the Plancherel formula $\langle f, g\rangle=\langle\mathrm{F}(f), \mathrm{F}(g)\rangle$ where $f \in \mathcal{D}\left(\mathbb{A}_{C}\right), g \in \mathcal{D}^{\prime}\left(\mathbb{A}_{C}\right)$ and $\langle-,-\rangle$ is the canonical pairing between dual spaces. When we apply this formula to the characteristic functions $\delta_{\mathbf{A}_{1}(D)}$ and $\delta_{K}$ the result easily yields Riemann-Roch theorem together with Serre duality for divisors on $C$ (see for example [58]).

Trying to extend the harmonic analysis to the higher local fields and adelic groups we meet the following obstacle. The $n$-dimensional local fields and consequently the adelic groups are not locally compact topological groups for $n>1$ in any reasonable sense whereas by a theorem of Weil the existence of Haar measure (in the usual sense) on a topological group implies its local compactness. Unfortunately, the well-known extensions of this measure theory to the infinite-dimensional spaces or groups (such as the Wiener measure) do not help in our circumstances. Thus, we have to develop a measure theory and harmonic analysis on $n$-dimensional local fields and adelic groups $a b$ ovo.

The idea for dealing with this problem came to me in the 1990s. In dimension one, local fields and adelic groups are equipped with a natural filtration provided by fractional ideals $\wp^{n}, n \in \mathbb{Z}$, which correspond to the standard valuations. For example,

\footnotetext{
${ }^{4}$ This means that $f$ is a composition of an open epimorphism and a closed monomorphism.
} 
this filtration on the field $\mathbb{F}_{q}((t))$ is given by the powers of $t$. If $P \supset Q$ are two elements of such a filtration on a group $V$, then the Bruhat space $\mathcal{D}(V)$ is canonically isomorphic to the double inductive limit of the (finite-dimensional) spaces $\mathcal{F}(P / Q)$ of all functions on the finite groups $P / Q$. The other function spaces listed above can be represented in the same way if we use all possible combinations of projective or inductive limits.

In dimension two, local fields $K$ such as $K_{P, C}$ again have a filtration by fractional ideals, which are powers of $\wp$. But now, the quotient $P / Q=\wp^{m} / \wp^{n}, n>m$ will be isomorphic to a direct sum of finitely many copies of the residue field $\bar{K}=\mathbb{F}_{q}((u))$. Thus this group is locally compact and the functional space $\mathcal{D}(P / Q)$ is well defined. To define the function spaces on $K$ one can try to repeat the procedure which we know for the 1-dimensional fields. To do that, we need to define the maps (direct or inverse images) between the spaces $\mathcal{D}(P / Q), \mathcal{D}(P / R), \mathcal{D}(Q / R)$ for $P \supset Q \supset R$. The selection rules mentioned above restrict the opportunities for this construction. This enables us to introduce the following six types of spaces of functions (or distributions) on $V$ :

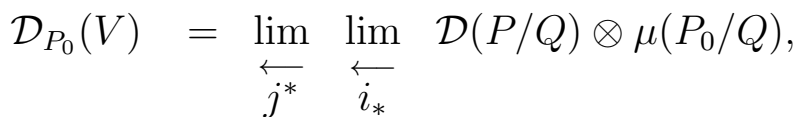

$$
\begin{aligned}
& \mathcal{D}^{\prime} P_{0}(V)=\lim _{\overrightarrow{j_{*}}} \underset{i^{*}}{\lim } \mathcal{D}^{\prime}(P / Q) \otimes \mu\left(P_{0} / Q\right)^{-1}, \\
& \mathcal{E}(V)=\lim _{j^{*}} \underset{i^{*}}{\lim } \mathcal{E}(P / Q) \\
& \mathcal{E}^{\prime}(V)=\lim _{\overrightarrow{j_{*}}} \underset{\lim _{*}}{ } \mathcal{E}^{\prime}(P / Q) \\
& \tilde{\mathcal{E}}(V)=\lim _{i^{*}} \underset{j^{*}}{\lim } \tilde{\mathcal{E}}(P / Q), \\
& \tilde{\mathcal{E}}^{\prime}(V)=\lim _{i_{*}} \underset{j_{*}}{\lim } \tilde{\mathcal{E}}^{\prime}(P / Q)
\end{aligned}
$$

where $P \supset Q \supset R$ are some elements of the filtration in $V$ (with locally compact quotients), $P_{0}$ is a fixed subgroup from the filtration and $j: Q / R \rightarrow P / R, i: P / R \rightarrow$ $P / Q$ are the canonical maps.

This definition works for a general class of groups $V$ including the adelic groups such as $\mathbb{A}_{X}$, which has a filtration by the subspaces $\mathbb{A}_{12}(D)$ where $D$ runs through the Cartier divisors on $X$.

Thus, developing of harmonic analysis may start with the case of dimension zero (finite-dimensional vector spaces over a finite field representing a scheme of dimension zero, such as $\operatorname{Spec}\left(\mathbb{F}_{q}\right)$, or finite abelian groups) and then be extended by induction to the higher dimensions.

An important contribution was made in 2001 by Michael Kapranov [33] who suggested using a trick from the construction of the Sato Grassmanian in the theory of 
integrable systems (known as a construction of semi-infinite monomials) 5 . The idea consists of using the spaces $\mu\left(P_{0} / Q\right)$ of measures instead of $\mu(P / Q)$ in the above definition of the spaces $\mathcal{D}_{P_{0}}(V)$ and $\mathcal{D}^{\prime}{ }_{P_{0}}(V)$ : without it one cannot define the functional spaces for all adelic groups in the two-dimensional case and, in particular, for the whole adelic space $\mathbb{A}_{X}$.

In 2005 Denis Osipov has introduced the notion of a $C_{n}$ structure in the category of filtered vector spaces [49]. With this notion at hand, harmonic analysis can be developed in a very general setting, for all objects of the category $C_{2}$. The crucial point is that the $C_{n}$-structure exists for the adelic spaces of any $n$-dimensional noetherian scheme. The principal advantage of this approach is that one can perform all the constructions simultaneously in the local and global cases. The category $C_{1}$ contains (as a full subcategory) the category of linearly locally compact vector spaces (introduced and thoroughly studied by S. Lefschetz [42]) and there one can use the classical harmonic analysis.

When we go to general arithmetic schemes over $\operatorname{Spec}(\mathbb{Z})$, fields like $\mathbb{C}\left(\left(t_{1}\right)\right) \ldots\left(\left(t_{n}\right)\right)$ appear and we need to extend the basic category $C_{n}$. In dimension one, this means that connected Lie groups must also be considered. It is possible to define categories of filtered abelian groups $C_{n}^{a r},(n=0,1,2)$, which contain all types of groups which arise from arbitrary schemes of dimension 0,1 and 2 (in particular from algebraic surfaces over $\mathbb{F}_{q}$ and arithmetic surfaces). Harmonic analysis can be developed for these categories if we introduce function spaces which are close to that of classical functional analysis, such as Schwartz space $\mathcal{S}(\mathbb{R})$ of smooth functions on $\mathbb{R}$, which are rapidly decreasing together with all their derivatives. Recall that in the case of dimension one we had to consider, in addition to the genuine local fields such as $\mathbb{Q}_{p}$, the fields $\mathbb{R}$ and $\mathbb{C}$. In the next dimension, we have to add to the two-dimensional local fields such as $\mathbb{F}_{q}((u))((t))$ or $\mathbb{Q}_{p}((t))$ the fields $\mathbb{R}((t))$ and $\mathbb{C}((t))$. They will occupy the entire row in the table above. This theory has been developed in papers [50, 51].

Just as in the case of dimension one, we define direct and inverse images in the categories of groups, which take into account all the components of the adelic complex, the Fourier transform $\mathrm{F}$ which preserves the spaces $\mathcal{D}$ and $\mathcal{D}^{\prime}$ but interchanges the spaces $\mathcal{E}$ and $\mathcal{E}^{\prime}$. We also introduce the characteristic functions $\delta_{W}$ of subgroups $W$ and then prove a generalization of the Poisson formula. It is important that for a certain class of groups $V$ (but not for $\mathbb{A}_{X}$ itself) there exists a nonzero invariant measure, defined up to multiplication by a constant, which is an element of $\mathcal{D}^{\prime}(V)$. Another important tool of the theory are the base change theorems for the inverse and direct images. They are function-theoretic counterparts of the classical base change theorems in the categories of coherent sheaves.

The applications of the theory includes an analytic expression for the intersection number of two divisors based on an adelic approach to the intersection theory [55] and an analytic proof of the (easy part of) Riemann-Roch theorem for divisors on $X$.

This theory is the harmonic analysis on the additive groups of the local fields and adelic rings (including their archimedean cousins). In the classical case of dimension one, the analysis can be developed on arbitrary varieties (defined either over $K$, or over

\footnotetext{
${ }^{5} \mathrm{~A}$ construction of this kind for the local fields is also contained in $\mathbf{3 5}$.
} 
A). This has already been done by Bruhat in the local case [10. For arbitrary varieties defined over a two-dimensional local field $K$, this kind of analysis was carried out by D. Gaitsgory and D. A. Kazhdan in [18 for the purposes of representation theory of reductive groups over the field $K$. This was preceded by a construction [34 of harmonic analysis on homogenous spaces such as $G(K) / G\left(O_{K}^{\prime}\right)$ (introduced in [56]). We note that the construction of harmonic analysis (over $K$ and $\mathbb{A}$ ) is a nontrivial problem even in the case $G=\mathbb{G}_{m}$. This will be the topic of our discussion in the following sections.

\section{Discrete Adelic Groups on Two-dimensional Schemes}

The harmonic analysis discussed above can be viewed as a representation theory of the simplest algebraic group over local or adelic rings, namely, of the additive group. In general, 1-dimensional local fields and adelic rings lead to a vastly developed representation theory of reductive groups over these fields and rings. The simplest case of this theory is still the case of an abelian group, namely GL(1). Let $K$ be a local field of dimension 1 . Then $\mathrm{GL}(1, K)=K^{*}$, the multiplicative group of $K$, and the irreducible representations are the abelian characters, i.e. continuous homomorphisms $\chi: K^{*} \rightarrow \mathbb{C}^{*}$. For arithmetic applications one requires the morphisms to $\mathbb{C}^{*}$, not to the unitary group $\mathbb{U}(1) \subset \mathbb{C}^{*}$.

The 1 -dimensional local field $K$ contains a discrete valuation subring $\mathcal{O}$ with a maximal ideal $\wp$. Then the local group $K^{*}$ has the following structure

$$
K^{*}=\left\{t^{n}, n \in \mathbb{Z}\right\} \times \mathcal{O}^{*}=\left\{t^{n}, n \in \mathbb{Z}\right\} \times \bar{K}^{*} \times\{1+\wp\},
$$

where $t$ is a generator of the ideal $\wp, \bar{K}=\mathbb{F}_{q}$ and the group $\{1+\wp\}$ is the projective limit of its finite quotients $\{1+\wp\} /\left\{1+\wp^{n}\right\}$. Thus, our group $K^{*}$ is a product of the maximal compact subgroup $\mathcal{O}^{*}$ and a discrete group $\cong \mathbb{Z}$. When $K$ is the local field $K_{P}$ attached to a point $P$ of an algebraic curve $C$ defined over a finite field $\mathbb{F}_{q}$, let us set $\Gamma_{P}:=K_{P}^{*} / \mathcal{O}_{P}^{*}$. In the adelic case, we set

$$
\Gamma_{C}:=\mathbf{A}_{C}^{*} / \prod_{P} \mathcal{O}_{P}^{*}=\bigoplus_{P} K_{P}^{*} / \mathcal{O}_{P}^{*}=\bigoplus_{P} \mathbb{Z}
$$

This group is the group of divisors on $C$.

We now introduce the groups dual to these discrete groups viewing them as algebraic groups defined over $\mathbb{C}$ :

$$
\mathbb{T}_{P}=\operatorname{Hom}\left(\Gamma_{P}, \mathbb{C}^{*}\right), \quad \mathbb{T}_{S}=\prod_{P \in S} \mathbb{T}_{P}, \quad \mathbb{T}_{C}=\underset{S}{\lim } \mathbb{T}_{S}
$$

where $S$ runs through all finite subsets in $C$. Let us consider the divisor $D_{S}$ with normal crossings on $\mathbb{T}_{S}$ that consists of the points in the product $\mathbb{T}_{S}$ for which at least one component is the identity point in some $\mathbb{T}_{P}$. Let $\mathbb{C}_{+}\left[\mathbb{T}_{S}\right]$ be the space of rational functions on $\mathbb{T}_{S}$ that are regular outside $D_{S}$ and may have poles of first order on $D_{S}$. The space $\mathbb{C}_{+}\left[\mathbb{T}_{C}\right]$ can be defined as an inductive limit with respect to the obvious inclusions. 
We would like to show that harmonic analysis on the adelic space $\mathbb{A}_{C}$ can be reformulated in terms of complex analysis on the dual groups. We need one more torus $\mathbb{T}_{0} \cong \mathbb{C}^{*}$, which corresponds by the duality to the image of the degree map

$$
\operatorname{deg}: \Gamma_{C} \rightarrow \mathbb{Z} \quad \text { with } \operatorname{deg}(D)=\sum_{P} n_{P} \operatorname{deg}(P) \text { for a divisor } D=\sum_{P} n_{P} P \text {. }
$$

Denote by $j: \mathbb{T}_{0} \rightarrow \mathbb{T}_{C}$ the natural embedding. Then the following diagram

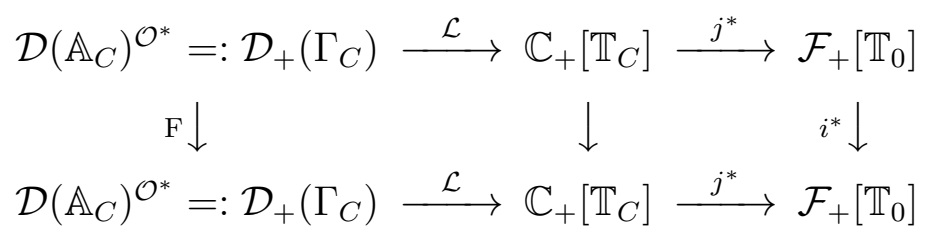

commutes. Here, the map $\mathrm{F}$ is induced by the Fourier transform on the adelic group $\mathbb{A}_{C}$, the map $i: \mathbb{T}_{0} \rightarrow \mathbb{T}_{0}$ sends $z \in \mathbb{T}_{0}$ to $q^{-1} z^{-1}$ and the space $\mathcal{F}_{+}\left[\mathbb{T}_{0}\right]$ consists of the functions that are regular outside the points $z=1$ and $z=q^{-1}$ and may have poles of the first order at these points. We denoted here by $\mathcal{L}$ a duality map, a version of the Fourier transform in this situation (completely different however from the Fourier map F). If $g \in G$ and $z \in \mathbb{T}_{G}=\operatorname{Hom}\left(G, \mathbb{C}^{*}\right)$ for some group $G$ then $(\mathcal{L} f)(z)=\sum_{g} f(g) z(g)$.

The next important fact is a reformulation of the Poisson formula on the group $\mathbb{A}_{d} 6$. It can be shown that for any function $f \in \mathcal{D}\left(\mathbb{A}_{C}\right)^{\mathcal{O}^{*}}$

$$
\begin{gathered}
\sum_{\gamma \in K} f(\gamma)=\operatorname{res}_{(0)}(\omega)+\operatorname{res}_{(1)}(\omega), \\
\sum_{\gamma \in K}(\operatorname{Ff} f)(\gamma)=-\operatorname{res}_{\left(q^{-1}\right)}(\omega)-\operatorname{res}_{(\infty)}(\omega),
\end{gathered}
$$

where $\omega=j^{*} \mathcal{L} f d z / z$ is the differential form on the compactification of the torus $\mathbb{T}_{0}$ and the points we have chosen for the residues are $z=0, z=q^{-1}, z=1$ and $z=\infty$. Since the poles of the form $\omega$ are contained in this set, we deduce that the Poisson formula on the curve $C$ (with an appropriate choice of Haar measure on $\mathbb{A}_{C}$ ) is equivalent to the residue formula (4) for the form $\omega$ on the compactification of the torus $\mathbb{T}_{0}$ (the general case see in 62 6 ).

Our main goal now is to understand what correspond to these constructions in the case of dimension twd7. Let us first consider the local situation, that is we fix a flag $P \in C$ on $X$ and assume, for the sake of simplicity, that $P$ is a smooth point on $C$. The local field $K_{P, C}$ has the discrete valuation subring $\widehat{\mathcal{O}}_{P, C}$. It is mapped onto the local field $k(C)_{P}$ on $C$. This local field contains his own discrete valuation subring $\widehat{\mathcal{O}}_{P}$ and we denote its preimage in $\widehat{\mathcal{O}}_{P, C}$ by $\widehat{\mathcal{O}}_{P, C}^{\prime}$ We set

$$
\Gamma_{P, C}:=K_{P, C}^{*} / \widehat{\mathcal{O}}_{P, C}^{\prime *}
$$

\footnotetext{
${ }^{6}$ For the sake of simplicity, we assume that $\operatorname{Pic}^{0}(C)\left(\mathbb{F}_{q}\right)=(0)$, that is $\operatorname{Ker}(\operatorname{deg})=\operatorname{Div}_{l}(C)$.

${ }^{7} \mathrm{We}$ consider here the case of an algebraic surface. The main definitions remain valid for the scheme part of an arithmetic surface.
} 
where $\Gamma_{P, C}$ is a certain abelian group, which is (non-canonically) isomorphic to $\mathbb{Z} \oplus \mathbb{Z}$. However, there is a canonical exact sequence of abelian groups

$$
0 \rightarrow \mathbb{Z} \rightarrow \Gamma_{P, C} \rightarrow \mathbb{Z} \rightarrow 0
$$

The map to $\mathbb{Z}$ in the sequence corresponds to the discrete valuation $\nu_{C}$ with respect to $C$ and the subgroup $\mathbb{Z}$ corresponds to the discrete valuation $\nu_{P}$ on $C$ at $P$. A choice of local coordinates $u, t$ in a neighborhood of $P$ such that locally $C=\{t=0\}$ provides a splitting of this exact sequence. The group $\Gamma_{P, C}$ will then be isomorphic to the subgroup $\left\{t^{n} u^{m}, n, m \in \mathbb{Z}\right\}$ in $K_{P, C}^{*}$.

The group of coordinate transformations $u \mapsto u, t \mapsto u^{k} t, k \in \mathbb{Z}$ preserves extension (11). Therefore, this determines an embedding

$$
\mathbb{Z} \rightarrow \operatorname{Aut}\left(\Gamma_{P, C}\right)
$$

which in fact is canonical.

We are now going to produce a global analogue of the local construction given above. For that purpose, consider the subgroup $\widehat{\mathcal{O}}^{\prime *}$ of $\mathbb{A}_{X}^{*}$, defined as the adelic product of the local groups $\widehat{\mathcal{O}}_{P, C}^{\prime *}$ for all flags on an algebraic surface $X$. Let us consider the quotient

$$
\Gamma_{X}:=\mathbb{A}_{X}^{*} / \widehat{\mathcal{O}}^{\prime *}=: \prod_{(P, C)}{ }^{\prime} \Gamma_{P, C}
$$

We have a natural surjective homomorphism $\mathbb{A}_{X}^{*} \rightarrow \Gamma_{X}$ and all subgroups in $\mathbb{A}_{X}^{*}$ such as $\mathbb{A}_{01}^{*}, \mathbb{A}_{12}^{*}, \ldots, \mathbb{A}_{0}^{*}$ have their images $\Gamma_{01}, \Gamma_{12}, \ldots, \Gamma_{0}$ in $\Gamma_{X}$.

Then the structure of $\Gamma_{X}$ can be described by an exact sequence

$$
0 \rightarrow \prod_{C} \operatorname{Div}(C) \longrightarrow \Gamma_{X} \stackrel{\pi}{\longrightarrow} \bigoplus_{C} \prod_{P \in C}^{\prime} \mathbb{Z} \rightarrow 0
$$

where, as above, $\operatorname{Div}(C)$ denotes the group of divisors on a curve $C \subset X$ and the restricted product $\prod^{\prime} \mathbb{Z}$ denotes the set of collections of integers with components whose absolute values are bounded. More precisely,

(1) The subgroups $\prod_{C} \operatorname{Div}(C)$ and $\Gamma_{12}$ in $\Gamma_{X}$ coincide.

(2) The restriction of the homomorphism $\pi$ to the subgroup $\Gamma_{02} \subset \Gamma_{X}$ is an isomorphism:

$$
\left.\pi\right|_{\Gamma_{02}}: \Gamma_{02} \stackrel{\sim}{\longrightarrow} \bigoplus_{C} \prod_{P \in C}{ }^{\prime} \mathbb{Z}
$$

In other words, we see that there is a canonical splitting $\Gamma_{X}=\Gamma_{12} \oplus \Gamma_{02}$ of exact sequence (13) which is independent of any possible choice of the coordinates. The groups which we have constructed are abelian. In our two-dimensional case, the crucial point is that they are provided with certain canonical central extensions. 
Let us start once more with the local situation, that is we fix a flag $P \in C$ on $X$. Following [2] (see also [30]) we have a canonical central extension of groups

$$
1 \rightarrow k(C)_{P}^{*} \rightarrow \tilde{K}_{P, C}^{*} \rightarrow K_{P, C}^{*} \rightarrow 1
$$

such that the corresponding commutator map in the central extension is a skew form $\langle\cdot, \cdot\rangle: K_{P, C}^{*} \times K_{P, C}^{*} \rightarrow k(C)_{P}^{*}$ given by the tame symbol (without sign), that is by

$$
\langle f, g\rangle=f^{\nu_{C}(g)} g^{-\nu_{C}(f)}(\bmod \wp) \in k(C)_{P}^{*},
$$

where $\wp$ is the ideal which defines the curve $C$.

There exists a canonical section of extension (14) over the subgroup $\widehat{\mathcal{O}}_{P, C}^{\prime *} \subset K_{P, C}^{*}$. Denote by $\tilde{\mathcal{O}}_{P, C}^{\prime *}$ the image of $\widehat{\mathcal{O}}_{P, C}^{* *}$ in $\tilde{K}_{P, C}^{*}$ with respect to this section. If we take the quotient of the extension (14) by the subgroup $\widehat{\mathcal{O}}_{P}^{*}$ of the center $k(C)_{P}^{*}$ and then by the subgroup $\tilde{\mathcal{O}}_{P, C}^{\prime *}$ we obtain a new central extension

$$
0 \rightarrow \mathbb{Z} \rightarrow \tilde{\Gamma}_{P, C} \rightarrow \Gamma_{P, C} \rightarrow 0 .
$$

It is well known that $H^{2}(\mathbb{Z} \oplus \mathbb{Z}, \mathbb{Z})=\mathbb{Z}$ and the extension (16) is a generator of this group. The commutator in this central extension defines a non-degenerate symplectic form $\langle-,-\rangle$ on $\Gamma_{P, C}$ with values in $\mathbb{Z}$. Let us fix local parameters $u, t$ at $P$. Then $\Gamma_{P, C}$ is isomorphic to the group of matrices

$$
\left(\begin{array}{lll}
1 & n & c \\
0 & 1 & p \\
0 & 0 & 1
\end{array}\right)
$$

with integer entries and $\langle n, p\rangle=n p$. We denote this group by Heis $(3, \mathbb{Z})$. Hence, we arrive at the following class of discrete nilpotent groups.

Definition 2. Let $H, H^{\prime}$, and $\mathrm{C}$ be abelian groups and let $\langle-,-\rangle: H \times H^{\prime} \rightarrow \mathrm{C}$ be a biadditive pairing. The set $H \times H^{\prime} \times \mathrm{C}$ with the composition law $(n, p, c)(m, q, a)=$ $(n+m, p+q, c+a+\langle n, q\rangle)$, where $n, m \in H, p, q \in H^{\prime}$ and $c, a \in \mathrm{C}$, is called the discrete Heisenberg group $G$.

One then constructs the Heisenberg group $G$ as a group of upper triangular unipotent matrices with $H$ and $H^{\prime}$ on the second diagonal and $\mathrm{C}$ in the right top corner. There is the obvious natural central extension

$$
0 \rightarrow \mathrm{C} \rightarrow G \rightarrow H \oplus H^{\prime} \rightarrow 0
$$

In the global case, we have the Heisenberg group $\tilde{\Gamma}_{X}$ with

$$
\begin{gathered}
H=\Gamma_{12}=\prod_{C} \operatorname{Div}(C)=\prod_{C} \bigoplus_{P \in C} \mathbb{Z}, \quad H^{\prime}=\Gamma_{02} \cong \bigoplus_{C} \prod_{P \in C}{ }^{\prime} \mathbb{Z} \\
\mathrm{C}=I_{X}:=\bigoplus_{C} \bigoplus_{P \in C} \mathbb{Z}
\end{gathered}
$$


and the pairing $H \times H^{\prime} \rightarrow \mathbb{C}$ is given by a component-wise multiplication. We thus get a central extension

$$
0 \rightarrow I_{X} \rightarrow \tilde{\Gamma}_{X} \rightarrow \Gamma_{X} \rightarrow 0
$$

and for each flag $P \in C$ the restriction of extension (18) to $\Gamma_{P, C}$ coincides with extension (16). So, we obtain in this way a global analogue of the local construction, since we could describe $\tilde{\Gamma}_{X}$ as an "adelic" product of the local groups $\tilde{\Gamma}_{P, C}$ in an appropriate sense.

There is a natural surjective homomorphism $\varphi: I_{X} \rightarrow Z^{2}(X), \quad\left(n_{P, C}\right) \mapsto$ $\sum_{P}\left(\sum_{C \ni P} n_{P, C}\right)[P]$, where $Z^{2}(X)$ denotes the group of zero-cycles on $X$. We set

$$
I_{02}:=\operatorname{Ker}(\varphi), \quad I_{01}:=\bigoplus_{C} \operatorname{Div}_{l}(C) \subset \bigoplus_{C} \operatorname{Div}(C)=I_{X}
$$

The Heisenberg group $\tilde{\Gamma}_{X}$ is closely related to the main arithmetic groups attached to the surface $X$. The quotient $I_{X} /\left(I_{01}+I_{02}\right)$ is the second Chow group $C H^{2}(X)$ of $X$. Also, there are isomorphisms

$$
\begin{gathered}
\Gamma_{01} /\left(\Gamma_{0}+\Gamma_{1}\right) \cong\left(\Gamma_{12} \cap\left(\Gamma_{01}+\Gamma_{02}\right)\right) / \Gamma_{1} \cong \\
\cong\left(\Gamma_{02} \cap\left(\Gamma_{01}+\Gamma_{12}\right)\right) / \Gamma_{0} \cong \operatorname{Pic}(X) .
\end{gathered}
$$

Moreover, the pairing $\Gamma_{12} \times \Gamma_{02} \rightarrow I_{X}$ corresponds to the intersection pairing $\operatorname{Pic}(X) \times$ $\operatorname{Pic}(X) \rightarrow C H^{2}(X)$.

It is remarkable that the groups $K_{P, C}^{*}$ (and the global adelic groups), which are very far from being locally compact, nevertheless have a non-trivial discrete quotient.

\section{Representations of Discrete Heisenberg Groups}

We have seen that in the case of dimension two the first non-trivial nilpotent groups have occured. To define their duals one needs to develop an appropriate representation theory for this class of groups.

For the discrete groups the classical theory of unitary representations on a Hilbert space is not so well developed since these groups are mostly not of type I. By Thoma's theorem, a discrete group is of type I if and only if it has an abelian subgroup of finite index.

This implies a violation of the main principles of representation theory on Hilbert spaces: non-uniqueness of the decomposition into irreducible components; too bad topology of the unitary dual space; non-existence of characters... . V. S. Varadarajan wrote in 1989: "A systematic developement of von Neumann's ideas led eventually (in the 1950s) to a deep understanding of the decomposition of unitary representations and to results which implied more or less that a reasonable generalization of classical Fourier analysis and representation theory could be expected only for the so-called type I groups; i.e. groups all of whose factor representations are of type $I "[\mathbf{7 0}]$. 
We can also say that the class of unitary representations is too restrictive for the arithmetic purposes.

On the other hand, there exists a theory of smooth representations for $p$-adic algebraic groups. This theory is also valid for a more general class of totally disconnected locally compact groups. Discrete groups are a simple particular case of this class of groups and the general theory delivers a reasonable class of representations, namely representations on a vector space without any topology. The new viewpoint consists in a systematic consideration of purely algebraic representations in place of unitary representations on Hilbert spaces.

Following [63], we consider now this representation theory for the discrete Heisenberg groups $G=\left(H, H^{\prime}, \mathrm{C},\langle-,-\rangle\right)$ where all three groups are finitely generated. We introduce the complex tori $\mathbb{T}_{H}=\operatorname{Hom}\left(H, \mathbb{C}^{*}\right), \mathbb{T}_{H^{\prime}}=\operatorname{Hom}\left(H^{\prime}, \mathbb{C}^{*}\right)$ and $\mathbb{T}_{\mathrm{C}}=\operatorname{Hom}\left(\mathrm{C}, \mathbb{C}^{*}\right)$, and set $\mathbb{T}_{G}=\mathbb{T}_{H} \times \mathbb{T}_{H^{\prime}} \times \mathbb{T}_{\mathrm{C}}$. The group $H$ is homomorphically mapped to $\mathbb{T}_{H^{\prime}}$ according to the rule:

$$
h \in H \mapsto\left\{h^{\prime} \mapsto \chi_{\mathrm{C}}\left(\left\langle h, h^{\prime}\right\rangle\right)\right\} .
$$

Denote the kernel of this map by $H_{\chi}$. If $\chi \in \mathbb{T}_{H^{\prime}}$ then let $h(\chi)$ be the translate of the character $\chi$ by the image of $h$ in $\mathbb{T}_{H^{\prime}}$. We have $h\left(\chi_{H^{\prime}}\right)(p)=\chi_{H^{\prime}}(p) \chi_{\mathrm{C}}(\langle h, p\rangle)$ for any $p \in H^{\prime}$. For any $\chi \in \mathbb{T}_{G}, \chi=\chi_{H} \otimes \chi_{H^{\prime}} \otimes \chi_{C}$, let $G_{\chi}=H_{\chi} H^{\prime} \mathrm{C}$ be the subset in $G$. Then $G_{\chi}$ is a normal subgroup in $G$, which depends only on $\chi_{C}$ and $\chi \mid G_{\chi}$ is a character of the group $G_{\chi}[65]$.

Definition 3. Let $V_{\chi}$ be the space of all complex-valued functions $f$ on $G$ which satisfy the following conditions:

1. $f(h g)=\chi(h) f(g)$ for all $h \in G_{\chi}$.

2. The support $\operatorname{Supp}(f)$ is contained in the union of a finite number of cosets of $G_{\chi}$.

Right translations define a representation $\pi_{\chi}$ of the group $G$ on the space $V_{\chi}$. One can prove that these representations $\pi_{\chi}$ are irreducible in both possible senses: there are no nontrivial invariant subspaces, and the Schur lemma holds. Furthermore, these representations can be completely classified. Namely, the representations $V_{\chi}$ and $V_{\chi^{\prime}}$ are equivalent if and only if three following conditions are satisfied:

1. $\chi_{\mathrm{C}}=\chi_{\mathrm{C}}^{\prime}$.

2. There exists $h \in H$ such that $\chi_{H^{\prime}}^{\prime}=h\left(\chi_{H^{\prime}}\right)$.

3. $\chi_{H}^{\prime}(h)=\chi_{H}(h)$ for all $h \in H_{\chi}$ or equivalently there exists $t \in \mathbb{T}_{H / H_{\chi}}=$ $\operatorname{Hom}\left(H / H_{\chi}, \mathbb{C}^{*}\right) \subset \mathbb{T}_{H}$ such that $\chi_{H}^{\prime}=t\left(\chi_{H}\right)$.

Here the torus $\mathbb{T}_{H / H_{\chi}}$ acts on the ambient torus $\mathbb{T}_{H}$ by translations. The equivalence classes of representations $V_{\chi}$ therefore correspond to orbits of the groups $\mathbb{T}_{H / H_{\chi}} \times H / H_{\chi}$ in subsets $\mathbb{T}_{H} \times \mathbb{T}_{H^{\prime}} \times\left\{\chi_{C}\right\}$ of the torus $\mathbb{T}_{G}$.

The group $G$ is a semidirect product of the groups $H$ and $H^{\prime} C$ and the main tool for obtaining the results stated above is the Mackey formalism 43 . which describes the category of induced representations for semi-direct products of abelian locally compact 
groups. In the classical theory, this is well-known for unitary representations on Hilbert spaces. In our case, we can use the version of this formalism developed in the theory of representation of $p$-adic reductive groups $[4,15,71$.

The restriction of functions from the group $G$ to the subgroup $H$ defines a bijection of $V_{\chi}$ with a certain space of functions on $H$. This space has an explicit basis and we can now define the character of the representation $\pi_{\chi}$ as the matrix trace of the representation operators $\pi_{\chi}(g)$ with respect to this basis. It is easy to see that in many cases the corresponding infinite sum of diagonal elements will diverge. The simplest example is the group $\operatorname{Heis}(3, \mathbb{Z})$, see (17).

It is nevertheless possible to define the character if we apply a well-known construction from the theory of loop groups [64][ch. 14.1]. Namely, we have to add some "loop rotations " to the group $G$. In our context, this means that the group $G$ has to be extended to a semi-direct product $\hat{G}=G \rtimes A$, where $A \subset \operatorname{Hom}\left(H, H^{\prime}\right)$ is a non-trivial subgroup.

To construct the group $\hat{G}=G \rtimes A$, one needs to extend the automorphisms of the abelian groups $H \oplus H^{\prime}$ to the automorphisms of the entire Heisenberg group. Note that the group $A$ acts on $H \oplus H^{\prime}$ by unipotent transformations. When we fix an $r \in H$ and choose $k \in A$, the expression

$$
k(m, p, c)=(m, p+k(m), c+1 / 2\langle m-r, k(m)\rangle) \quad m \in H, p \in H^{\prime}, c \in \mathrm{C}
$$

defines an automorphism of the group $G$ if the following conditions hold:

1. $\left\langle m, k\left(m^{\prime}\right)\right\rangle=\left\langle m^{\prime}, k(m)\right\rangle$ for all $m, m^{\prime} \in H$

2. $\langle m-r, k(m)\rangle \in 2 \mathrm{C}$ for all $m \in H$.

In the case of the group $\Gamma_{P, C} \cong \operatorname{Heis}(3, \mathbb{Z})$, (see (17)), we have $A=\mathbb{Z}, r=1$ and $k \in A$ acts as $k(n, p, c)=\left(n, p+k n, c+\frac{1}{2} k n(n-1)\right), n, p, c \in \mathbb{Z}$. Then the extension is suggested by the existence of the group of coordinate transformations on the surface $X$ (see (12)) at the point $P$ :

$$
t \mapsto u^{k} t, u \mapsto u, k \in \mathbb{Z} .
$$

According to the analogy between algebraic and arithmetic surfaces we discussed above, these coordinate transformations in the two-dimensional local field $K_{P, C}=\mathbb{F}_{q}((u))((t))$ indeed correspond to the loop rotations

$$
t \mapsto \alpha t, \alpha \mapsto \alpha, \alpha \in \mathbb{C}
$$

in the field $\mathbb{C}((t)$ on an arithmetic surface.

When $k\left(H_{\chi}\right) \subset \operatorname{Ker}\left(\chi_{H^{\prime}}\right)$ the representation of $G$ on $V_{\chi}$ can be extended to a representation $\hat{\pi}_{\chi}$ of the extended group $\hat{G}$ on the same space. Let

$$
\left(\mathbb{T}_{\mathrm{C}} \times A\right)_{+}:=\left\{\chi \in \mathbb{T}_{\mathrm{C}}, k \in A:\left|\chi_{\mathrm{C}}(\langle n, k(n)\rangle)\right|<1 \text { for all } n \in H / H_{\chi}, n \neq 0\right\}
$$

be a relation in $\mathbb{T}_{\mathrm{C}} \times A$, let $A(\chi)$ be the projection of the set $\left(\mathbb{T}_{\mathrm{C}} \times A\right)_{+} \cap(\{\chi\} \times A)$ to $A$ and let $\hat{G}(\chi)=G \times A(\chi) \subset \hat{G}$. 
We can now solve the existence problem for the characters. The trace $\operatorname{Tr} \hat{\pi}_{\chi}(g)$ exists for all $g \in \hat{G}(\chi)$ and we have

$$
\operatorname{Tr} \hat{\pi}_{\chi}(g)=\chi_{H}(m) \chi_{H^{\prime}}(p) \chi_{\mathrm{C}}(c) \cdot \sum_{n \in H / H_{\chi}} \chi_{H^{\prime}}(k(n)) \chi_{\mathrm{C}}(\langle n, p\rangle+1 / 2\langle n-r, k(n)\rangle) .
$$

for $g=(m, p, c, k), k \in A(\chi), m \in H_{\chi}$. The trace is zero if $m$ does not belong to $H_{\chi}$.

The trace is well-defined, but does not determine a function on the set of equivalence classes of representations. To overcome this difficulty, we have to consider representations of the extended group $\hat{G}$.

Let $\mathbb{T}_{A}=\operatorname{Hom}\left(A, \mathbb{C}^{*}\right)$ and $\mathbb{T}_{\hat{G}}=\mathbb{T}_{G} \times \mathbb{T}_{A}$. If $\hat{\chi}=\left(\chi, \chi_{A}\right) \in \mathbb{T}_{\hat{G}}$, then we set

$$
\hat{\pi}_{\hat{\chi}}=\hat{\pi}_{\chi} \otimes \chi_{A}
$$

We therefore have $\operatorname{Tr} \hat{\pi}_{\hat{\chi}}=\operatorname{Tr} \hat{\pi}_{\chi} \cdot \chi_{A}$. For a given $g \in \hat{G}(\chi)$, the trace $\operatorname{Tr} \hat{\pi}_{\hat{\chi}}(g)$ can be considered as a function on the domain $T^{\prime}=\mathbb{T}_{H} \times \mathbb{T}_{H^{\prime}} \times \mathbb{T}_{\mathrm{C}}(k) \times \mathbb{T}_{A}$ in the torus $\mathbb{T}_{\hat{G}}$, where $\mathbb{T}_{\mathrm{C}}(k)$ is the projection of the set $\left(\mathbb{T}_{\mathrm{C}} \times A\right)_{+} \cap\left(\mathbb{T}_{\mathrm{C}} \times\{k\}\right)$ to the torus $\mathbb{T}_{\mathrm{C}}$.

Let us define an action of the group $\mathbb{T}_{H / H_{\chi}} \times H$ on the set $\mathbb{T}_{H} \times \mathbb{T}_{H^{\prime}} \times\left\{\chi_{\mathrm{C}}\right\} \times \mathbb{T}_{A} \subset T^{\prime}$ by the formula

$$
(t, h)\left(\chi_{H}, \chi_{H^{\prime}}, \chi_{\mathrm{C}}, \chi_{A}\right)=\left(t\left(\chi_{H}\right), h\left(\chi_{H^{\prime}}\right), \chi_{\mathrm{C}}, \chi_{A}^{\prime}\right),
$$

where

$$
\chi_{A}^{\prime}(k)=\chi_{A}(k) \chi_{H^{\prime}}(k(h)) \chi_{\mathrm{C}}(1 / 2\langle h-r, k(h)\rangle), k \in A .
$$

We define the space $\mathcal{M}_{G}(k), k \in A$ as the quotient of the domain $T^{\prime}$ by this action. The quotient-space is a complex-analytic manifold, in fact a fibration over a domain in $\mathbb{T}_{\mathrm{C}}$. For a given $g=(m, p, c, k) \in \hat{G}(\chi)$ the trace $\operatorname{Tr} \hat{\pi}_{\hat{\chi}}(g)$ is invariant, under a simple additional condition, under the action (20) and defines a holomorphic function $F_{g}=F_{g}(\hat{\chi})$ on $\mathcal{M}_{G}(k)$. We now obtain the main property that the characters must enjoy:

Let $\hat{\chi}, \hat{\chi}^{\prime} \in \mathbb{T}_{\hat{G}}$. The representations $\hat{\pi}_{\hat{\chi}}$ and $\hat{\pi}_{\hat{\chi}^{\prime}}$ are equivalent if and only if $\hat{G}(\chi)=$ $\hat{G}\left(\chi^{\prime}\right)$ and $F_{g}(\hat{\chi})=F_{g}\left(\hat{\chi}^{\prime}\right)$ for all $g \in \hat{G}(\chi)$.

Thus we see that the space $\mathcal{M}_{G}(k)$ is actually a moduli space for a certain class of representations of $\hat{G}$.

Let us consider the simplest example, that of the group Heis $(3, \mathbb{Z})$. Let $A=\mathbb{Z}=$ $\operatorname{Hom}\left(H, H^{\prime}\right), r=1, \hat{G}=G \rtimes \mathbb{Z}$ and $\chi_{\mathrm{C}}(c)=\lambda^{c}, \chi_{\mathrm{C}} \in \mathbb{T}_{\mathrm{C}}(k>0)$ where $\mathbb{T}_{\mathrm{C}}(k>0)=$ $\{0<|\lambda|<1\}$. Then $\mathbb{T}_{H^{\prime}} / \operatorname{Im} H=: E_{\lambda}$ is an elliptic curve, where $z \in \mathbb{T}_{H^{\prime}}=\mathbb{C}^{*}, \operatorname{Im} H=$ $\left\{\lambda^{\mathbb{Z}}\right\}$. We have a degree map

$$
\operatorname{Pic}\left(E_{\lambda}\right)=H^{1}\left(E_{\lambda}, \mathcal{O}^{*}\right)=H^{1}\left(H, \mathcal{O}^{*}\left(\mathbb{T}_{H^{\prime}}\right)\right) \rightarrow \operatorname{Hom}\left(H, H^{\prime}\right)=A
$$

and

$$
\operatorname{Pic}\left(E_{\lambda}\right)=\left\{\varphi(n, z)=a^{-n} z^{-k n} \lambda^{-1 / 2 k n(n-1)}: a \in \mathbb{C}^{*}, k \in A=\mathbb{Z}\right\} .
$$

Let $L$ be the line bundle which corresponds to a 1-cocycle $\varphi$. Then

$$
H^{0}\left(E_{\lambda}, L\right)=\left\{f(z), z \in \mathbb{T}_{H^{\prime}}: f\left(\lambda^{n} z\right)=\varphi(n, z) f(z)\right\}
$$


The theta-series

$$
\vartheta_{p, k, a}(z, \lambda):=z^{p} \sum_{n \in \mathbb{Z}} a^{n} z^{k n} \lambda^{n p+1 / 2 k n(n-1)}
$$

(which are the Poincaré series with respect to $\varphi$ ) converge for all $z \in \mathbb{C}^{*}, 0<|\lambda|<$ $1, k>0$, and form a basis of the space $H^{0}\left(E_{\lambda}, L\right)$ for $0 \leq p<k$. Finally,

$$
\operatorname{Tr} \hat{\pi}_{\hat{\chi}}(0, p, c, k)=\lambda^{c} t^{k} \vartheta_{p, k, 1}(z, \lambda),(z, \lambda) \in \mathcal{A}_{G}(k), t \in \mathbb{T}_{A}
$$

In this case, the theta-series lifted to $\overline{\mathcal{A}}_{G}(k)=\mathbb{C} \times\{$ upper halfplane $\}$ are Jacobi modular forms (up to some powers of $\lambda$ and $z$ ) with respect to the standard action of a finite index subgroup of the group $(\mathbb{Z} \oplus \mathbb{Z}) \rtimes \mathrm{SL}(2, \mathbb{Z}))$. This last statement is completely parallel to a well-known property of characters for representations of affine Kac-Moody algebras 31, 64.

In the more general situation in which $H$ and $H^{\prime}$ are torsion-free groups and $C=\mathbb{Z}$, $\left|\chi_{C}(c)\right| \neq 1$ for $c \neq 0$, the map $k: H \rightarrow H^{\prime}$ is a monomorphism with finite cokernel, $A=\mathbb{Z} k$ and the form $\langle-, k(-)\rangle$ is positive-definite, we have two dual abelian varieties $E=\mathbb{T}_{H^{\prime}} / \operatorname{Im} H$ and $E^{\prime}=\mathbb{T}_{H} / \operatorname{Im} H^{\prime}$ with the Poincaré bundle $\mathcal{P}$ over $E \times E^{\prime}$. The morphism $k$ defines an isogeny $\varphi_{k}: E \rightarrow E^{\prime}$ and the sheaf $L$ is defined as $\left(\operatorname{Id} \times \varphi_{k}\right)^{*} \mathcal{P}$. By Mumford's theory [44, there exists a finite Heisenberg group $\widetilde{\operatorname{Ker}}\left(\varphi_{k}\right)$, which is a central extension of the group $\operatorname{Ker}\left(\varphi_{k}\right)$. Then for all $g=(m, p, c, k) \in \hat{G}(\chi)$ the values of the characters $\operatorname{Tr} \hat{\pi}_{\hat{\chi}}(g) \chi_{\mathrm{C}}^{-1}(c) \chi_{A}^{-1}(k)$ are theta-functions for the bundle $L$.

If $\hat{\chi}=1 \otimes \chi_{H^{\prime}} \otimes \chi_{\mathrm{C}} \otimes 1$, then the functions $\operatorname{Tr} \hat{\pi}_{\hat{\chi}}(0, p, 0, k)$ for $p \in H^{\prime} \bmod k(H)$ form a basis of the space $H^{0}(E, L)$. This basis is a standard Mumford basis for the action of the Heisenberg group $\widehat{\operatorname{Ker}}\left(\varphi_{k}\right)=\left(H^{\prime} / H, \mathbb{T}_{H^{\prime} / H}, \mathbb{C}^{*}\right)$ on the space $H^{0}(E, L)$.

In addition, certain orthogonality relations are satisfied by the characters [63].

The boundary of the domain $\mathbb{T}_{\mathrm{C}}(k)$ can contain those characters $\chi_{0} \in \mathbb{T}_{\mathrm{C}}$ for which $H_{\chi_{0}}$ has a finite index in $H$. These characters correspond to the roots of unity in $\mathbb{C}^{*}$, so that the representations $\pi_{\chi_{0}}$ are finite-dimensional. Let $V=H \otimes \mathbb{R}$ and $Q$ be the extension of the pairing $\langle n, k(n)\rangle, n \in H$ to the space $V$. Also, let $\chi_{\mathrm{C}}(c)=\lambda^{c}$ and let us choose a boundary point $\chi_{0}$. The classical limit formulas for theta-functions imply the following behavior of the trace near the $\chi_{0}$ (we assume that $\chi_{H}=1$ and $\chi_{H}^{\prime}=1$ ):

$$
\operatorname{Tr} \hat{\pi}_{\hat{\chi}}(g) \sim \operatorname{Tr} \hat{\pi}_{\hat{\chi}_{0}}(g) \cdot\left[H: H_{\chi_{0}}\right]^{-1}\left(\operatorname{Det}_{V} Q\right)^{-1 / 2}\left(\frac{\sqrt{\pi}}{2}\right)^{\mathrm{rk} H} \log |\lambda|^{-\frac{1}{2} \mathrm{rk} H} \quad \text { when } \chi_{\mathrm{C}} \rightarrow \chi_{0} \text {. }
$$

The trace of the representation $\hat{\pi}_{\hat{\chi}_{0}}$ can be computed in terms of a Gauss sum.

Thus, we see that, in our situation, the change in the class of representations will cause the moduli spaces of induced representations to be complex-analytic manifolds. Characters do exist and are the modular forms. It seems that this more general holomorphic dual space is more adequate for this class of groups than the standard unitary dual which goes back to the Pontrjagin duality for abelian groups.

\section{$5 \quad$ Problems and Perspectives}

We collect here several problems related to the issues we have discussed in the talk. 


\section{Harmonic analysis for local fields and adelic groups of arbitrary dimen- sion $\mathbf{n}$.}

The basic category for this study has to be the category $C_{n}[49$ and its version that includes fields of the archimedean type [51]. When one tries to extend the measure theory and harmonic analysis to $n$-dimensional local fields and adelic groups for $n>2$ the following problem arises. The selection rules become too severe to go further in a straightforward way. This obstacle appears already for $n$-dimensional local fields with $n=3$. We can define the spaces analogous to $\mathcal{D}(V)$ or $\mathcal{D}^{\prime}(V)$ only under some strong restrictions on the groups $V\left(=\right.$ objects in $\left.C_{n}\right)$. Note that spaces such as $\mathcal{E}(V)$ can be easily defined for any $n$ and arbitrary group $V$.

\section{The Tate-Iwasawa method for two-dimensional schemes.}

J. Tate [68] and independently K. Iwasawa [29] reformulated the classical problem of analytic continuation for zeta- and L- functions for the fields of algebraic numbers and the fields of algebraic functions in one variable over a finite field. They introduced a new type of $L$-functions:

$$
L(s, \chi, f)=\int_{\mathbb{A}^{*}} f(g) \chi(g)|g|^{s} d^{*} g
$$

where $d^{*} g$ is a Haar measure on $\mathbb{A}^{*}$, the function $f$ belongs to the Bruhat-Schwartz space of functions on $\mathbb{A}_{X}$ and $\chi$ is an abelian character of the group $\mathbb{A}^{*}$ associated to a character

$$
\chi: \operatorname{Gal}\left(K^{\mathrm{ab}} / K\right) \rightarrow \mathbb{C}^{*}
$$

of the Galois group by the reciprocity map $\mathbb{A}^{*} \rightarrow \operatorname{Gal}\left(K^{\mathrm{ab}} / K\right)$. They also proved the analytic continuation of $L(s, \chi, f)$ to the entire $s$-plane and the functional equation

$$
L(s, \chi, f)=L\left(1-s, \chi^{-1}, \mathrm{~F}(f)\right)
$$

by means of the Fourier transform $\mathrm{F}$ and the Poisson formula for functions on $\mathbb{A}_{X}(8)$, (91).

For a special choice of $f$ and $\chi=1$ we obtain the zeta-function

$$
\zeta_{X}(s)=\prod_{x \in X}\left(1-(\# k(x))^{-s}\right)^{-1}
$$

of any scheme $X$ of dimension one (to which we have to add, if necessary, the archimedean factors). Here $x$ runs through the closed points of $X$. The product converges for $\operatorname{Re}(s)>$ $\operatorname{dim} X$.

There exists a general Hasse-Weil conjecture [23, 73] which asserts that these zeta(and more general $L$-) functions can be meromorphically extended to the entire $s$-plane and satisfy the functional equation (for regular proper schemes $X$ of dimension $n$ ) of the type $\zeta_{X}(n-s)=\{$ elementary factors $\} \zeta_{X}(s)$.

This conjecture has been completely proved for algebraic varieties defined over a finite field $\mathbb{F}_{q}$. For this goal the powerful machinery of the étale cohomology has been developed by A. Grothendieck. For schemes over $\operatorname{Spec}(\mathbb{Z})$, the general results are known only in dimension one, thanks to the Hecke's theorem. Later this was included into 
the Tate-Iwasawa approach. At the same time, this approach works for algebraic curves defined over $\mathbb{F}_{q}$. For the higher dimensions over $\operatorname{Spec}(\mathbb{Z})$, there are only scattered results; however these include the proof of the Hasse-Weil conjecture for elliptic curves over $\mathbb{Q}$ [75, 8].

For a long time the author has advocated the following

Problem. Extend Tate-Iwasawa's analytic method to higher dimensions (see in particular [58]).

The higher adeles were introduced precisely for this purpose. We hope that harmonic analysis and representation theory of adelic groups on two-dimensional schemes may help to solve this problem.

\section{Behavior of zeta- and L-functions in the critical strip.}

The critical strip for the ordinary Riemann's zeta-function is $0 \leq \Re(s) \leq 1$ and this zeta-function (with an archimedean factor) has there exactly two poles, both of first order. For the two-dimensional case, the critical strip is wider, namely $0 \leq \Re(s) \leq 2$. Take as $X$ a model over $\operatorname{Spec}(\mathbb{Z})$ of an elliptic curve $E$ defined over $\mathbb{Q}$. The Birch and Swinnerton-Dyer conjecture [5, 69] states that

$$
\zeta_{X}(s) \underset{s \rightarrow 1}{\sim} \frac{\# E(\mathbb{Q})_{\text {tor }}^{2}}{c \Omega \operatorname{Det}_{E(\mathbb{Q})}\langle-,-\rangle \# \amalg}(s-1)^{-r-2},
$$

where $E(\mathbb{Q})$ is the finitely generated Mordell-Weil group of rational points on $E, r$ is its rank, $\langle-,-\rangle$ is the height pairing, $\Omega$ is the real period of the curve, $\amalg$ is the ShafarevichTate group and $c$ is a product of certain local invariants.

Many years ago several people, including the author, have independently observed that this limit behavior is very similar to the limit behavior of a theta-function attached to a lattice. Namely, let $V / \mathbb{R}$ be a finite dimensional euclidean vector space of dimension $n$. Denote by $\langle-,-\rangle$ the scalar product on $V$. Let $\Gamma$ be a finitely generated abelian group such that $\Gamma \otimes \mathbb{R}=V$ and let $\Gamma^{\prime}=\Gamma / \Gamma_{\text {tor }}$ be the corresponding lattice (= a discrete co-compact subgroup) in $V$. Then the theta-function $\theta_{\Gamma}(t)$ is defined as

$$
\theta_{\Gamma}(t):=\sum_{\gamma \in \Gamma} e^{-\pi t\langle\gamma, \gamma\rangle}=\# \Gamma_{t o r} \cdot \theta_{\Gamma^{\prime}}(t)
$$

and satisfies the functional equation

$$
\theta_{\Gamma^{\prime}}(t)=t^{-\frac{n}{2}} \operatorname{Vol}\left(\Gamma^{\prime}\right)^{-1} \theta_{\Gamma^{\prime \perp}}\left(t^{-1}\right),
$$

where $\Gamma^{\perp} \subset V$ is the dual lattice and the volume of the fundamental domain for $\Gamma^{\prime}$ is $\operatorname{Vol}\left(\Gamma^{\prime}\right)=\operatorname{det}\left(\left\langle e_{i}, e_{j}\right\rangle\right)^{1 / 2}$ with $\left\{e_{i}\right\}$ a basis of the free $\mathbb{Z}$-module $\Gamma^{\prime}$.

In particular, we get

$$
\theta_{\Gamma}(t) \underset{t \rightarrow 0}{\sim} \# \Gamma_{t o r} \operatorname{Vol}(\Gamma)^{-1} t^{-\frac{n}{2}}
$$

If we apply this asymptotic formula to the group $\Gamma \oplus \Gamma$ then we get

$$
\theta_{\Gamma \oplus \Gamma}(t) \underset{t \rightarrow 0}{\sim} \frac{\# \Gamma_{t o r}^{2}}{\operatorname{Det}_{\Gamma}\langle-,-\rangle} t^{-n}
$$


which looks rather similar to the conjecture (23) if we take as $\Gamma$ the group $E(\mathbb{Q}) \oplus \mathbb{Z} \oplus \mathbb{Z}$. D. Zagier has devoted to this relation a note [77] with many interesting remarks and observations. In particular, he discussed the question of interpreting such factors as $\Omega$ and \#W which are not visible in the theta-formula (24).

In order to clarify the situation, let us look at the corresponding behavior of the zeta-function of an algebraic surface $X$ defined over $\mathbb{F}_{q}$. The analogy between geometric surfaces over $\mathbb{F}_{q}$ and arithmetic surfaces such as this model $X$ of $E$ suggests that this may be a useful move.

The value of the zeta function at $s=1$ is given by the conjecture of Artin and Tate [69, 45]. We assume that $X$ is a smooth proper irreducible surface. Denote by $\rho=\operatorname{rk} \mathrm{NS}(X)$ the rank of the Neron-Severi-group of $X$ and let $\left\{D_{i}\right\}$ with $D_{i} \in \operatorname{NS}(X)$ $i=1, \ldots, \rho$ be a basis of $\in \mathrm{NS}(X) \otimes \mathbb{Q}$. Denote by $D_{i} \cdot D_{j}$ their intersection index. Let $\operatorname{Br}(X)=H^{2}\left(X_{e t}, \mathcal{O}_{X}\right)$ be the Brauer group of $X$. Then the group $\operatorname{Br}(X)$ is conjectured to be finite and the following relation holds:

$$
\zeta_{X}(s) \underset{s \rightarrow 1}{\sim}(-1)^{\rho-1} q^{\chi\left(\mathcal{O}_{X}\right)} \frac{\# \operatorname{Pic}(X)_{\text {tor }}^{2}}{\# H^{0}\left(X, \mathcal{O}_{X}^{*}\right)^{2} \# \operatorname{Br}(X) \operatorname{det}\left(\left(D_{i} \cdot D_{j}\right)\right)}\left(1-q^{1-s}\right)^{-\rho} .
$$

Within the framework of the analogy between geometry and arithmetic [61, the group $\operatorname{NS}(X)$ corresponds to the group $E(\mathbb{Q}) \oplus \mathbb{Z} \oplus \mathbb{Z}$, the intersection index corresponds to the height pairing, the period $\Omega$ corresponds to $q^{\chi\left(\mathcal{O}_{X}\right)}$ and the Brauer group to the Shafarevich-Tate group $\amalg$.

Since $\left(1-q^{1-s}\right)^{-\rho} \underset{s \rightarrow 1}{\sim}(s-1)^{-\rho}(\log q)^{-\rho}$, we again guess that certain theta-functions related to the lattice $\mathrm{NS}(X)$ may have this kind of the limit behavior. An immediate objection to this suggestion is that the intersection pairing is not positive-definite. This can be resolved if we consider the Siegel theta-functions attached to indefinite quadratic forms.

The case of surfaces makes it clear that this question is highly non-trivial. Zetafunctions of algebraic varieties over $\mathbb{F}_{q}$ are very simple analytic functions. Indeed, according to Grothendieck's theory, they are equal to $F\left(q^{-s}\right)$ where $F(t)$ is a rational function of a variable $t$. The theta-functions involved are certainly transcendental functions, which cannot be simplified in this way by substitution. Thus the problem we arrive at is to understand how theta-functions can appear in this setting in a natural way, and how to relate them to zeta-functions. We conjecture that the theta-functions which occur into the traces of representations of the adelic groups constructed above could be such theta-functions. Their behavior in the limit (22) has the structure we have just described.

It is worth mention another problem, the so called $S$-duality conjecture, which is quite close to what have been discussed here. The problem came from the quantum field theory [72] but has purely algebraic formulation for an algebraic surface $X$ over a finite field $\mathbb{F}_{q}$ (see a discussion in [32]). Let $M_{r, n}$ be a moduli space of semi-stable vector bundles $E$ on $X$ with given rank $r$, trivial determinant and the second Chern class $c_{2}(E)=n$. Then the formal series

$$
\sum_{n} \# M_{r, n}\left(\mathbb{F}_{q}\right) q^{-n s}
$$


is expected to have under mild conditions on $X$ a modular behavior with respect to a congruence subgroup of the group $\operatorname{SL}(2, \mathbb{Z})$. It is remarkable that the transcendental functions appear once more in relation to a surface defined over a finite field.

\section{Representations of discrete nilpotent groups.}

i) The representations $\pi_{\chi}$ and $\hat{\pi}_{\hat{\chi}}$ of the discrete Heisenberg groups are particular examples of the irreducible representations of these groups. Thus, the problem of classification of all irreducible representations arises. Of course, one needs to impose certain conditions in order to get a reasonable answer. In the theory of unitary representations for discrete nilpotent finitely generated groups $G$ on a Hilbert space such a condition was found in [9]. One says that a representation $\pi$ of $G$ on a space $V$ has the finite multiplicity property if there exists a subgroup $H \subset G$ which preserves a line $l$ in $V$ and such that the character of $H$ defined by the action of $H$ on $l$ occurs in $\left.\pi\right|_{H}$ as a discrete direct summand with finite multiplicity. Then the class of irreducible representations with this property coincides with the class of irreducible monomial (= induced by an one-dimensional character) representations of $G$.

It is highly desirable to define in our algebraic situation a class of "basic" induced representations which will play the role that the Verma modules or representations with highest weight do for the representations of reductive Lie groups (or algebraic groups). This is closely related to a problem of classification of (say, left) maximal ideals in the group ring of $G$.

ii) The moduli spaces $\mathcal{M}_{G}(k)$ defined above are orbit spaces for group actions. This construction looks very similar to the Kirillov's orbit method for connected real (or complex) nilpotent Lie groups $G$ (or nilpotent algebraic groups over $\mathbb{Q}_{p}$ ) [39] where the unitary dual is the space $\mathfrak{g}^{*} / G$ of co-adjoint orbits in the dual $\mathfrak{g}^{*}$ of the Lie algebra $\mathfrak{g}$ of $G$. Attempts to extend Kirillov's method to finitely generated nilpotent groups were made in [24, 36] (see also [6]). It seems that there is a general functorial definition of spaces such as $\mathcal{M}_{G}(k)$ for arbitrary nilpotent discrete groups which will replace the spaces $\mathfrak{g}^{*} / G$ in this situation, just as the torus $\mathbb{T}_{\hat{G}}$ may be an analogue of the space $\mathfrak{g}^{*}$. The Kirillov's character formula may also exist in this situation.

iii) When one tries to apply the representation theory developed in section 5 to the nilpotent groups which arise from the algebraic surfaces $X$ (section 4), one immediately observes that:

1) the groups like $\tilde{\Gamma}_{X}$ are not finitely generated;

2) the groups like $(\operatorname{Pic}(\mathrm{X}), \operatorname{Pic}(\mathrm{X}), \mathrm{CH}(\mathrm{X}))$ are equipped with the indefinite form $\langle-,-\rangle$.

Certainly, the representation theory cannot be automatically extended to the case of infinitely generated groups. In our case, the "big" group $\tilde{\Gamma}_{X}$ is the adelic product of simplest Heisenberg groups $\tilde{\Gamma}_{P, C}$ and consequently is an inductive limit of finite products of these local groups. We can easily extend all the representation-theoretic constructions to the case of $\tilde{\Gamma}_{X}$ if we apply the technique from the theory of adelic products of reductive algebraic groups over 1-dimensional local fields. The role of the compact subgroups is now played by co-finite products of the local Heisenberg groups.

The problem 2) can also be solved. A solution is based on using the Siegel thetafunctions for indefinite quadratic forms that are well suited for this situation. 
iv) An important problem is to develop an analysis on discrete Heisenberg groups $G$, in particular, to define appropriate function spaces on $G$, the analogue of the map $\mathcal{L}$ (see (8) in section 3) and to obtain a Plancherel-type theorem which relates the function spaces on $G$ and spaces of holomorphic (or meromorphic) functions on $\mathcal{M}_{G}(k)$.

v) There exists a general question of the decomposition into the irreducible components of representations of discrete nilpotent groups. It is known that the regular representation (on the $L^{2}$-space on $G$ ) of a discrete group $G$ may have very different decompositions into irreducible components (see a first example of this kind in [43]). On the other hand, in our situation there is a rather concrete problem: how does one decompose the natural fundamental representation of the group $\tilde{\Gamma}_{X}$ (and locally of the groups $\left.\tilde{\Gamma}_{P, C}\right)$ on the spaces $\mathcal{D}_{\mathbb{A}_{12}}\left(\mathbb{A}_{X}\right)^{\mathcal{O}^{\prime *}}$ or $\mathcal{D}_{\mathbb{A}_{12}}^{\prime}\left(\mathbb{A}_{X}\right)^{\mathcal{O}^{\prime *}}\left(\right.$ respectively in $\mathcal{D}_{\mathcal{O}_{P, C}}\left(K_{P, C}\right)^{\mathcal{O}_{P, C}^{\prime *}}$ or $\left.\mathcal{D}_{\mathcal{O}_{P, C}}^{\prime}\left(K_{P, C}\right)^{\mathcal{O}_{P, C}^{\prime *}}\right)$ on a surface $X$ ?

vi) Our theory deals with the discrete "part" of the adelic group $\mathbb{A}_{X}^{*}=\mathrm{GL}\left(1, \mathbb{A}_{X}\right)$. D. Gaitsgory and D. Kazhdan have extended the traditional theory of representations for reductive $p$-adic groups (parabolic induction, Jacquet functor, cuspidal representations) to the case of groups $\operatorname{GL}(n, K)$ where $K$ is a two-dimensional local field (and of more general reductive groups) [18, 19, 20. An important and certainly very hard problem is to merge these two theories, at least for the group $\mathrm{GL}\left(2, \mathbb{A}_{X}\right)$.

vii) For the schemes of dimension two, we constructed discrete Heisenberg groups, which are nilpotent groups of class 2 . It is possible to associate certain discrete adelic groups to schemes of arbitrary dimension $n$ and that are the nilpotent groups of class $n$.

In this text, we mainly gave a review of certain recent advances in the higher adelic theory. During the last thirty years, this theory was developed in many different directions. We finish with a short list of these developement:8 :

- residues and symbols [53, 54, 17, 76, 11, 12, 13, 37, 38, 47, 67, 52,

- class field theory for higher dimensions: the author, K. Kato and his school, S. V. Vostokov and his school, see surveys [17, 16, 28, 66.

- adelic resolutions for sheaves, intersection theory, Chern classes, Lefschetz formula for coherent sheaves [55, 76, 26, 27, 21, 22,

- algebraic groups over local fields, buildings, Hecke algebras [56, 60, 34, 18, 19, 20, 7 ]

- restricted adelic complexes and the Krichever correspondence [59, 46, 48, 40, 41]

- relations with non-commutative algebra [57, 78].

\section{References}

[1] Algebraic Number Theory, Ed. J. W. S. Cassels and A. Fröhlich, Academic Press, London-New York, 1967.

\footnotetext{
${ }^{8}$ This list of references does not pretend to be complete.
} 
[2] E. Arbarello, V. G. Kac, C. De Concini, The Infinite Wedge Representation and the Reciprocity Law for Algebraic Curves, Comm. Math. Phys., 117 (1988), 1-36.

[3] A. A. Beilinson, Residues and Adeles, Func. Anal. Appl., 14, no. 1 (1980), 34-35.

[4] J. N. Bernstein, A. V. Zelevinsky, Representations of the group $G L(n, F)$, where $F$ is a local non-archimedian field, Russian Math. Surveys, 31(1976), 1-68.

[5] B. Birch, H. P. F. Swinnerton-Dyer, Notes on elliptic curves I, II , Journ. Reine u. Angew. Math. 212 (1963), 7-25; 218 (1965), 79-108.

[6] Mitya Boyarchenko, Maria Sabitova, The orbit method for profinite groups and padic analogue of Brown's theorem, e-print arXiv: mathRT/0608126.

[7] A. Braverman, D. Kazhdan, Some examples of Hecke algebras for two-dimensional local fields, Nagoya Math. J., 184 (2006), 57-84.

[8] C. Breuil, B. Conrad, F. Diamond, R. Taylor, On the modularity of elliptic curves over $\mathbb{Q}$ : wild 3-adic exercises, J. Amer. Math. Soc., 14 (2001), no. 4, 843-939.

[9] I. D. Brown, Representation of finitely generated nilpotent groups, Pacif. J. Math., 45 (1973), 13-25.

[10] F. Bruhat, Distributions sur un groupe localement compact et applications à l'étude des représentations des groupes §-adiques, Bull. Soc. Math. France, 89 (1961), 4375 .

[11] J.-L. Brylinski, D. A. McLaughlin, Multidimensional reciprocity laws, J. Reine Angew. Math. 481 (1996), 125-147.

[12] J.-L. Brylinski, D. A. McLaughlin, Characteristic classes and multidimensional reciprocity laws, Math. Res. Lett. 3 (1996), No.1, 19-30.

[13] J.-L. Brylinski, D. A. McLaughlin, The geometry of two-dimensional symbols, KTheory 10 (1996), No.3, 215-237.

[14] M. Burger, Analyse Harmonique sur les groupes de Heisenberg généralisés, Monatsch. für Math., 98 (1984), 29-40.

[15] P. Cartier, Representations of $\wp$-dic groups: a survey, Automorphic forms, representations and $L$-functions, Proc. Symposia in Pure Math., 33 (1979), Part 1, Amer. Math. Soc., Providence, RI, 1979, 111-155.

[16] I. B. Fesenko, S. V. Vostokov, Local fields and their extensions. A constructive approach, Amer. Math. Soc., Providence, RI, 1993, 283pp.

[17] T. Fimmel, A. N. Parshin, An introduction to the higher adelic theory, preprint 1999. 
[18] D. Gaitsgory, D. Kazhdan, Representations of algebraic groups over a 2-dimensional local field, Geom. and Funct. Analysis, 14 (2004), n 3, 535-574; arXiv math. $\mathrm{RT} / 0302.174$.

[19] D. Gaitsgory, D. Kazhdan, Algebraic groups over a 2-dimensional local field: irreducibility of certain induced representations, J. Differ. Gom. 70 (2005), No. 1, 113-127 (2005); arXiv: math/0409543.

[20] D. Gaitsgory, D. Kazhdan, Algebraic groups over a 2-dimensional local field: some further constructions, Bernstein, Joseph (ed.) et al., Studies in Lie theory. Dedicated to A. Joseph on his sixtieth birthday. Progress in Mathematics 243, Basel: Birkhauser, 2006, 97-130; arXiv: math/0406282.

[21] S. O. Gorchinskiy, A. N. Parshin, Adelic Lefschetz formula for the action of a onedimensional torus, Uraltseva, N.N.(ed.), Proceedings of the St. Petersburg Mathematical Society. Vol. XI. . Translations. Series 2. Amer. Math. Soc. 218 (2006), 31-48; e-print arXiv: math/0408058.

[22] S. O. Gorchinskiy, An adelic resolution for homology sheaves, Izvestiya: Mathematics 72 (2008), No. 6, 1187-1252; e-print arXiv: math/0705.2597.

[23] H. Hasse Zetafunktionen und L-Funktionen zu einem arithmetischen Funktionenkrper vom Fermatschen Typus, Abh. S. Akad. Wiss. Berlin Math. Kl. (1954), 570 (= Helmut Hasse, Mathematische Abhandlungen, Band 2, Berlin-New York: Walter de Gruyter, 1975, S. 450).

[24] R. E. Howe, On representations of discrete, finitely generated, torsion-free, nilpotent groups Pacif. J. Math. 73 (1977), 281-305.

[25] A. Huber, On the Parshin-Beilinson Adeles for Schemes, Abh. Math. Sem. Univ. Hamburg 61 (1991), 249-273.

[26] R. Hübl, A. Yekutieli, Adeles and differential forms, J. Reine u. Angew. Math. 471 (1996), 1-22.

[27] R. Hübl, A. Yekutieli, Adelic Chern forms and applications, Am. J. Math. 121 (1999), No.4, 797-839.

[28] Invitation to higher local fields (Münster, 1999), (ed. I. Fesenko, M. Kurihara), Geom. Topol. Monogr., 3, Geom. Topol. Publ., Coventry, 2000, 304pp.

[29] K. Iwasawa, A note on functions, Proc. Intern. Congres Math. (1950), Cambridge, Mass., 1950, vol.2, 322.

[30] V. G. Kac, D. H. Peterson, Spin and wedge representations of infinite-dimensional Lie algebras and groups, Proc. Nat. Acad. Sci. USA 78 (1981), 3308-3312.

[31] V. G. Kac, D. H. Peterson, Infinite-dimensional Lie algebras, Theta-functions and Modular Forms, Advances in Math. 53(1984), 125-264. 
[32] M. M. Kapranov, The elliptic curve in the S-duality theory and Eisenstein series for Kac-Moody groups, e-print arXiv:mathAG/0001005.

[33] M. M. Kapranov, Semiinfinite symmetric powers, e-print arXiv: math.QA/0107089.

[34] M. M. Kapranov, Double affine Hecke algebras and 2-dimensional local fields, J. Amer. Math. Soc., 14 (2001), n 1, 239-262; e-print arXiv math.AG/9812021.

[35] D. A. Kazhdan, Fourier transform over local fields, Milan J. Math. 74 (2006), 213225.

[36] K. P. Kokhas', Finite factor representations of 2-step nilpotent groups and orbit theory, Zap. Nauchn. Semin. POMI 307, 120-140, 282 (2004); translation in J. Math. Sci., New York 131 (2005), No. 2, 5508-5519.

[37] A. G. Khovanskii, An analog of determinant related to Parshin-Kato theory and integer polytopes, Funct. Anal. Appl. 40 (2006), No. 2, 126-133.

[38] A. G. Khovanskii, Logarithmic functional and reciprocity laws, Toric topology: international conference, May 2008, Contemporary Mathematics, Vol. 46 (2008).

[39] A. A. Kirillov, Lectures on the orbit method, Graduate Studies in Mathematics, vol. 64, Providence, RI: Amer. Math.Soc. 2004, xx, 408 p.

[40] H. Kurke, D. V. Osipov, A. B. Zheglov, Formal punctured ribbons and twodimensional local fields, J. Reine u. Angew. Math. 629 (2009) , 133-170; e-print arXiv: math/07080985.

[41] H. Kurke, D. V. Osipov, A. B. Zheglov, Formal groups arising from formal punctured ribbons, Intern. J. Math. 21 (2010), 755-797. to appear; e-print arXiv:0901.1607.

[42] S. Lefschetz, Algebraic topology, AMS Colloquium Publications 27, Amer. Math. Soc. New York, 1942.

[43] G. Mackey, On induced representations of groups, Amer. J. Math. 73 (1951), 576592.

[44] D. Mumford, On equations defining abelian varieties I, Inv. Math., 1 (1966), 287354.

[45] J. S. Milne, On a conjecture of Artin and Tate, Ann. of Math. 102(1975), 517-533.

[46] D. V. Osipov, The Krichever correspondence for algebraic varieties, Izvestiya. Mathematics 65(2001), no. 5, 941-975; arXiv: math/0003188.

[47] D. V. Osipov, Central extensions and reciprocity laws on algebraic surfaces, Sbornik: Mathematics 196, N 10 (2005), 1503-1527; see also arXiv: math.NT/0501155. 
[48] D. V. Osipov, n-dimensional local fields and adeles on $n$-dimensional schemes, Surveys in Contemporary Mathematics, Edited by N. Young, Y. Choi; London Mathematical Society Lecture Note Series, vol. 347 (2007), pp. 131-164, Cambridge University Press; e-print math.AG/0508205.

[49] D. V. Osipov, Adeles on $n$-dimensional schemes and categories $C_{n}$, Intern. J. Math., 18 (2007), no. 3 , 269-279; e-print arXiv: math.AG/0509189.

[50] D. V. Osipov, A. N. Parshin, Harmonic analysis on local fields and adelic spaces I, Izvestiya: Mathematics, 72 (2008), 915-976; e-print arXiv: math/0707.1766.

[51] D. V. Osipov, A. N. Parshin, Harmonic analysis on local fields and adelic spaces II, Izvestiya: Mathematics (to appear); e-print arXiv: math/0912.1577.

[52] Denis Osipov, Xinwen Zhu, Categorical proof of Parshin reciprocity laws on algebraic surface, e-print arXiv: math.AG/1002.4848.

[53] A. N. Parshin, Class fields and algebraic K-theory, Uspekhi Mat. Nauk. 30 (1975), 253-254 (in russian).

[54] A. N. Parshin, On the arithmetic of two-dimensional schemes I. Repartitions and residues, Math. USSR Izvestiya 10 (1976), No.4, 695-729.

[55] A. N. Parshin, Chern classes, adeles and L-functions, J. Reine u. Angew. Math., 341 (1983), 174-192.

[56] A. N. Parshin, Vector Bundles and Arithmetical Groups I, Proc. Steklov Inst. Math. 208 (1995), 212-233; e-print arXiv: alg-geom/9605001.

[57] A. N. Parshin, On a ring of formal pseudodifferential operators, Proc. Steklov Inst. Math. 224 (1999), 266-280; e-print arXiv: math/9911098.

[58] A. N. Parshin, Higher-dimensional local fields and L-functions, Invitation to higher local fields (Münster, 1999), 199-213, Geom.Topol. Monogr., 3, Geom. Topol. Publ., Coventry, 2000; e-print ArXiv: math.AG/0012151.

[59] A. N. Parshin, Integrable systems and local fields, Commun. Algebra 29(2001), No.9, 4157-4181; e-print ArXiv: math.AG/0912.1520.

[60] A. N. Parshin, Vector Bundles and Arithmetical Groups II, Proc. Steklov Inst. Math. 241 (2003), 179-191.

[61] A. N. Parshin, Numbers as functions: the development of an idea in the Moscow school of algebraic geometry, Bolibruch, A.A. (ed.) et al., Mathematical events of the twentieth century. Berlin: Springer; Moscow: Phasis., 2006, 297-329; e-print arXiv: math/0912.3785. 
[62] A. N. Parshin, Notes on the Poisson formula (Lectures on Representations of Adelic groups. Part I), Saint-Petersburg, POMI, November 2009, and Moscow, MIAN, December 2009, to appear in Saint-Petersburg Mathematical Journal; e-print arXiv: math/1011.3392.

[63] A. N. Parshin, On holomorphic representations of discrete Heisenberg groups, Funct. Anal. Appl., 44 (2010), N 2, 92-96.

[64] A. Pressley, G. Segal, Loop groups, Oxford, 1986.

[65] T. Pytlik, $L^{1}$-harmonic Analysis on Semi-Direct Products of Abelian groups, Mh. Math., 93 (1982), 309-328.

[66] W. Raskind, Abelian class field theory of arithmetic schemes, (Jacob, Bill (ed.) et al.), $K$-theory and algebraic geometry: connections with quadratic forms and division algebras. Proc. Symp. Pure Math. 58, Part 1, Amer. Math. Soc., Providence, RI, 1995, 85-187.

[67] I. Soprounov, Residues and tame symbols on toroidal varieties, e-print arXiv:math $\mathrm{AG} / 0203114$.

[68] J. Tate, Fourier analysis in number fields and Hecke's zeta-function, Thesis, Princeton, 1950 (published in [1]).

[69] J. Tate, On the conjectures of Birch and Swinnerton-Dyer and a geometric analog, Séminaire Bourbaki, 1965/66, exp. 306, 26pp.

[70] V. S. Varadarajan, An introduction to Harmonic analysis on semisimple Lie groups, Cambridge University Press, 1989.

[71] M.-F. Vignéras, Représentations l-modulaires d'un groupe réductif p-adique avec $l \neq p$, Birkhäuser, 1996.

[72] C. Vafa, E. Witten, A strong coupling test of S-duality, Nucl. Phys. B 431 (1994), 3-77.

[73] A. Weil, Number theory and algebraic geometry, Proc. Intern. Congr. Math.(Cambridge, 1950), vol. 2, 90-110 (= André Weil. Collected Papers,Vol. 1 (1926-1951). Heidelberg-Berlin:Springer, 1980 , 442-452.

[74] A. Weil, Basic Number Theory, Springer-Verlag, Berlin, 1967.

[75] A. J. Wiles, Modular elliptic curves and Fermat's last theorem, Ann. of Math. (2) 141 (1995), no. 3, 443-551.

[76] A. Yekutieli, An explicit construction of the Grothendieck residue complex, Astérisque, 208, 1992. 
[77] D. Zagier, The Birch-Swinnerton-Dyer conjecture from a naive point of view, Arithmetic Algebraic Geometry, Birkhäuser, Basel, 1991, 377-390.

[78] A. B. Zheglov, On wild division algebras over field of power series, Sbornik: Mathematics, 195 (2004), No. 6, 783-817. 